\title{
THE Q/U IMAGING EXPERIMENT: POLARIZATION MEASUREMENTS OF THE GALACTIC PLANE AT 43 AND $95 \mathrm{GHz}$
}

\author{
T. M. Ruud ${ }^{1}$, U. Fuskeland ${ }^{1}$, I. K. Wehus $^{2}$, M. Vidal ${ }^{3}$, D. Araujo ${ }^{4}$, C. BischofF ${ }^{5,6}$, I. Buder ${ }^{5,6}$, Y. Chinone ${ }^{7,8}$, K. Cleary ${ }^{9}$, \\ R. N. Dumoulin ${ }^{4}$, A. Kusaka ${ }^{10,11}$, R. Monsalve ${ }^{12}$, S. K. Næsss ${ }^{1,13}$, L. B. Newburgh ${ }^{14}$, R. A. Reeves ${ }^{15}$, J. T. L. Zwart ${ }^{4,16,17}$, \\ L. Bronfman $^{18}$, R. D. Davies ${ }^{3}$, R. Davis ${ }^{3}$, C. Dickinson ${ }^{3}$, H. K. Eriksen ${ }^{1}$, T. Gaier ${ }^{2}$, J. O. Gundersen $^{19}$, M. Hasegawa ${ }^{7}$, \\ M. Hazumi ${ }^{7}$, K. M. Huffenberger ${ }^{20}$, M. E. Jones ${ }^{13}$, C. R. Lawrence ${ }^{2}$, E. M. Leitch ${ }^{2}$, M. Limon ${ }^{4}$, A. D. Miller ${ }^{4}$, \\ T. J. Pearson ${ }^{9}$, L. Piccirillo ${ }^{3}$, S. J. E. Radford ${ }^{9}$, A. C. S. Readhead ${ }^{9}$, D. Samtleben ${ }^{21,22}$, M. Seiffert ${ }^{2}$, M. C. Shepherd ${ }^{9}$, \\ S. T. Staggs ${ }^{11}$, O. TAJima ${ }^{5,7}$, AND K. L. ThOMPSON ${ }^{23}$ \\ QUIET COLLABORATION \\ ${ }^{1}$ Institute of Theoretical Astrophysics, University of Oslo, P.O. Box 1029 Blindern, N-0315 Oslo, Norway; t.m.ruud@astro.uio.no \\ ${ }^{2}$ Jet Propulsion Laboratory, California Institute of Technology, 4800 Oak Grove Drive, Pasadena, CA 91109, USA \\ ${ }^{3}$ Jodrell Bank Centre for Astrophysics, Alan Turing Building, School of Physics and Astronomy, The University of Manchester, \\ Oxford Road, Manchester M13 9PL, UK \\ ${ }^{4}$ Department of Physics and Columbia Astrophysics Laboratory, Columbia University, New York, NY 10027, USA \\ ${ }^{5}$ Kavli Institute for Cosmological Physics, Department of Physics, Enrico Fermi Institute, The University of Chicago, Chicago, IL 60637, USA \\ ${ }_{7}^{6}$ Harvard-Smithsonian Center for Astrophysics, 60 Garden Street MS 42, Cambridge, MA 02138, USA \\ ${ }^{7}$ High Energy Accelerator Research Organization (KEK), 1-1 Oho, Tsukuba, Ibaraki 305-0801, Japan \\ Department of Physics, University of California, Berkeley, CA 94720, USA \\ ${ }^{9}$ Cahill Center for Astronomy and Astrophysics, California Institute of Technology, 1200 E. California Boulevard M/C 249-17, Pasadena, CA 91125, USA \\ ${ }^{10}$ Physics Division, Lawrence Berkeley National Laboratory, 1 Cyclotron Road, Berkeley, CA 94720, USA \\ ${ }^{11}$ Joseph Henry Laboratories of Physics, Jadwin Hall, Princeton University, Princeton, NJ 08544, USA \\ ${ }^{12}$ School of Earth and Space Exploration, Arizona State University, 781 E. Terrace Road, Tempe, AZ 85287, USA \\ ${ }_{13}^{13}$ Department of Astrophysics, University of Oxford, Keble Road, Oxford OX1 3RH, UK \\ ${ }^{14}$ Dunlap Institute, University of Toronto, 50 St. George Street, Toronto, ON M5S 3H4 \\ ${ }_{16}^{15}$ CePIA, Departamento de Astronomía, Universidad de Concepción, Casilla 160-C, Concepción, Chile \\ ${ }^{16}$ Physics Department, University of the Western Cape, Private Bag X17, Bellville 7535, South Africa \\ ${ }^{17}$ Astrophysics, Cosmology \& Gravity Centre, Department of Astronomy, University of Cape Town, Private Bag X3, Rondebosch 7701, South Africa \\ ${ }^{18}$ Departamento de Astronomía, Universidad de Chile, Casilla 36-D, Santiago, Chile \\ ${ }^{19}$ Department of Physics, University of Miami, 1320 Campo Sano Drive, Coral Gables, FL 33146, USA \\ ${ }^{20}$ Florida State University, Physics, Tallahassee, FL 32306, USA \\ ${ }^{21}$ Huygens-Kamerlingh Onnes Laboratorium, Universiteit Leiden, The Netherlands \\ ${ }^{22}$ Nikhef, Science Park, Amsterdam, The Netherlands \\ ${ }^{23}$ Stanford University and Kavli Institute for Particle Astrophysics and Cosmology, Stanford, CA 94305 USA \\ Received 2015 June 19; accepted 2015 July 31; published 2015 September 24
}

\begin{abstract}
We present polarization observations of two Galactic plane fields centered on Galactic coordinates $(l, b)=\left(0^{\circ}, 0^{\circ}\right)$ and $\left(329^{\circ}, 0^{\circ}\right)$ at both $\mathrm{Q}(43 \mathrm{GHz})$ and $\mathrm{W}$ bands $(95 \mathrm{GHz})$, covering between 301 and 539 square degrees depending on frequency and field. These measurements were made with the QUIET instrument between 2008 October and 2010 December, and include a total of $1263 \mathrm{hr}$ of observations. The resulting maps represent the deepest large-area Galactic polarization observations published to date at the relevant frequencies with instrumental rms noise varying between 1.8 and $2.8 \mu \mathrm{K} \mathrm{deg}, 2.3-6$ times deeper than corresponding WMAP and Planck maps. The angular resolution is $27 ! 3$ and 12 !. FWHM at Q and W bands, respectively. We find excellent agreement between the QUIET and WMAP maps over the entire fields, and no compelling evidence for significant residual instrumental systematic errors in either experiment, whereas the Planck $44 \mathrm{GHz}$ map deviates from these in a manner consistent with reported systematic uncertainties for this channel. We combine QUIET and WMAP data to compute inverse-variance-weighted average maps, effectively retaining small angular scales from QUIET and large angular scales from WMAP. From these combined maps, we derive constraints on several important astrophysical quantities, including a robust detection of polarized synchrotron spectral index steepening of $\approx 0.2$ off the plane, as well as the Faraday rotation measure toward the Galactic center $\left(\mathrm{RM}=-4000 \pm 200 \mathrm{rad} \mathrm{m}^{-2}\right)$, all of which are consistent with previously published results. Both the raw QUIET and the co-added QUIET+WMAP maps are made publicly available together with all necessary ancillary information.
\end{abstract}

Key words: cosmic background radiation - cosmology: observations - Galaxy: center - Galaxy: general polarization

\section{INTRODUCTION}

The field of cosmic microwave background (CMB) cosmology has undergone an important transition during the last two years. Until 2013, the primary limitation of most CMB experiments, whether targeting temperature or polarization fluctuations, was instrumental noise. Contamination from astrophysical foregrounds and systematic errors was generally small compared to the instrumental noise level or intrinsic cosmic variance of the observations, and only minor corrections for either were required to produce robust cosmological results. Three examples among many are CBI (Readhead et al. 2004; Sievers et al. 2007), BOOMERanG (MacTavish et al. 2006), and WMAP (Bennett et al. 2013). In this noise- 
dominated regime, the $\mathrm{CMB}$ field as a whole made rapid progress for more than two decades, with each new experiment improving cosmological parameter constraints in accordance with its respective noise level. Eventually, this process led to the current highly successful $\Lambda \mathrm{CDM}$ "concordance" cosmological model, which today describes virtually all currently available cosmological observations with only six free parameters (Bennett et al. 2013; Planck Collaboration XVI 2014; Planck Collaboration XIII 2015).

This situation changed dramatically with the Planck release in 2013 March (Planck Collaboration I 2014), and later with the BICEP2 release the following year (BICEP2 Collaboration et al. 2014). The exquisite instrumental sensitivity of Planck resulted in a CMB temperature likelihood that is, for the first time, limited by confusion from astrophysical foregrounds rather than instrumental noise (Planck Collaboration XV 2014; Planck Collaboration XI 2015). Likewise, BICEP2 was the first CMB B-mode polarization experiment to become foregroundlimited in polarization (BICEP2/Keck Array \& Planck Collaborations 2015).

To continue rapid progress toward a more refined cosmological model, in particular, with respect to large-scale polarization, reionization, and inflation (e.g., Liddle \& Lyth 2000, and references therein), a thorough understanding of relevant astrophysical foregrounds is paramount. Great progress has already been made on this (e.g., Finkbeiner et al. 1999; de Oliveira-Costa et al. 2008; Bennett et al. 2013; Ichiki 2014), and in early 2015 the Planck Collaboration presented the most detailed full-sky model for the frequency range between 30 and $353 \mathrm{GHz}$ to date, including both polarized synchrotron and thermal-dust emission over the full sky (Planck Collaboration X 2015). According to this model, the frequency minimum for polarized foregrounds on degree angular scales occurs between 70 and $80 \mathrm{GHz}$, varying only weakly with multipole range, probably depending somewhat on sky location.

In order to improve on this foreground model, better measurements are required with respect to both depth and frequency coverage. In addition, control of instrumental systematic errors is of course critical. As described in Planck Collaboration I (2015), Planck Collaboration II (2015), Planck Collaboration VIII (2015), and Planck Collaboration X (2015), there are still outstanding issues with the most recent Planck polarization observations, both below and above the foreground frequency minimum at $70 \mathrm{GHz}$. Cross-checks and comparisons with external data sets, including WMAP, can be helpful in identifying such issues. Other data sets anticipated in the very near future that should be useful in the effort to map out the foregrounds include S-PASS $(2.3 \mathrm{GHz}$; Carretti et al. 2009), C-BASS (5 GHz; King et al. 2010, 2014), and QUIJOTE (10-40 GHz; Rubiño-Martín et al. 2012), all observing at low frequencies.

In this paper, we present data that fit naturally into this larger astrophysical foreground program: measurements at 43 and $95 \mathrm{GHz}$ of two fields in the Galactic plane taken by the QUIET instrument (QUIET Collaboration et al. 2012b) between 2008 October and 2010 December. QUIET was a pathfinder experiment designed to improve limits on B-mode polarization and demonstrate the low level of systematic error achievable through the combination of careful monolithic-microwaveintegrated-circuit (MMIC) receiver module design, instrument design, and survey strategy. The instrument employed detector arrays comprising $19 \mathrm{Q}$ band $(43 \mathrm{GHz})$ and $90 \mathrm{~W}$ band $(95 \mathrm{GHz})$ detector modules, observing from the Atacama Desert in Chile. The experiment reported the cleanest microwave polarization spectra with respect to instrumental systematic errors at the time. The sum of all instrumental systematic errors was constrained to correspond to a tensor-toscalar ratio of $r \lesssim 0.01$ (QUIET Collaboration et al. 2012b). This result was only barely surpassed by the very recent and vastly more sensitive BICEP2 observations, which reported an equivalent limit on instrumental systematic errors of $r \lesssim 0.006$ (BICEP2 Collaboration et al. 2015). Cosmological CMB $E$ and $B$ angular power spectra were reported in QUIET Collaboration et al. (2011, 2012a), while constraints on polarized point sources were reported by QUIET Collaboration et al. (2014).

The rest of this paper is organized as follows. In Section 2, we review the QUIET data selection and processing pipeline as applied to the Galactic plane analysis, emphasizing those steps that are different compared to the original CMB-oriented analysis. We discuss the Q-band maps derived for the Galactic center field in Section 3, while equivalent discussions and figures for the remaining observations are deferred to the appendix. In Section 4, we derive constraints on important astrophysical quantities such as the spectral index of synchrotron emission and the Faraday rotation measure toward the Galactic center, both of which are critical for performing robust astrophysical component separation. We summarize and conclude in Section 5. All final data products (sky maps, mask, noise covariance matrices, and beam profiles) are available on the LAMBDA website. $^{24}$ Following both WMAP and Planck, we adopt the HEALPix (Gorski et al. 2005) convention for polarization, which differs from the IAU convention in the sign of the Stokes $U$ parameter. All maps are provided in Galactic coordinates.

\section{OBSERVATIONS AND DATA PROCESSING}

The basic data selection and processing steps were described in detail in QUIET Collaboration et al. (2011, 2012a). We briefly review the main steps in the following, describing in greater detail a few notable differences between the previous and the updated analysis. The most important of these is coaddition with a second experiment (typically WMAP), which is essential in order to robustly measure angular scales comparable to the size of the observed field. While CMB power spectrum or cosmological parameter estimation can be done without them, these modes are essential for deriving astrophysical spectral parameters, such as the synchrotron spectral index or thermal dust temperature. A second difference is in the data-selection criteria, which are slightly less stringent in this Galactic analysis than in the original CMB analysis because the signal-to-noise ratio is higher for the Galactic fields.

In the original QUIET analysis work, two pipelines were developed independently for cross-validation purposes, one implementing a pseudo- $C_{\ell}$ framework (Hivon et al. 2002; QUIET Collaboration et al. 2011), and the other implementing a brute-force maximum-likelihood framework (Tegmark 1997; Bond et al. 1998; QUIET Collaboration et al. 2011). A major advantage of the latter is that it provides both unbiased sky maps and corresponding dense pixel-pixel covariance matrices, which are useful for comparison and inverse-noise-variance co-

\footnotetext{
${ }^{24}$ http://lambda.gsfc.nasa.gov
} 


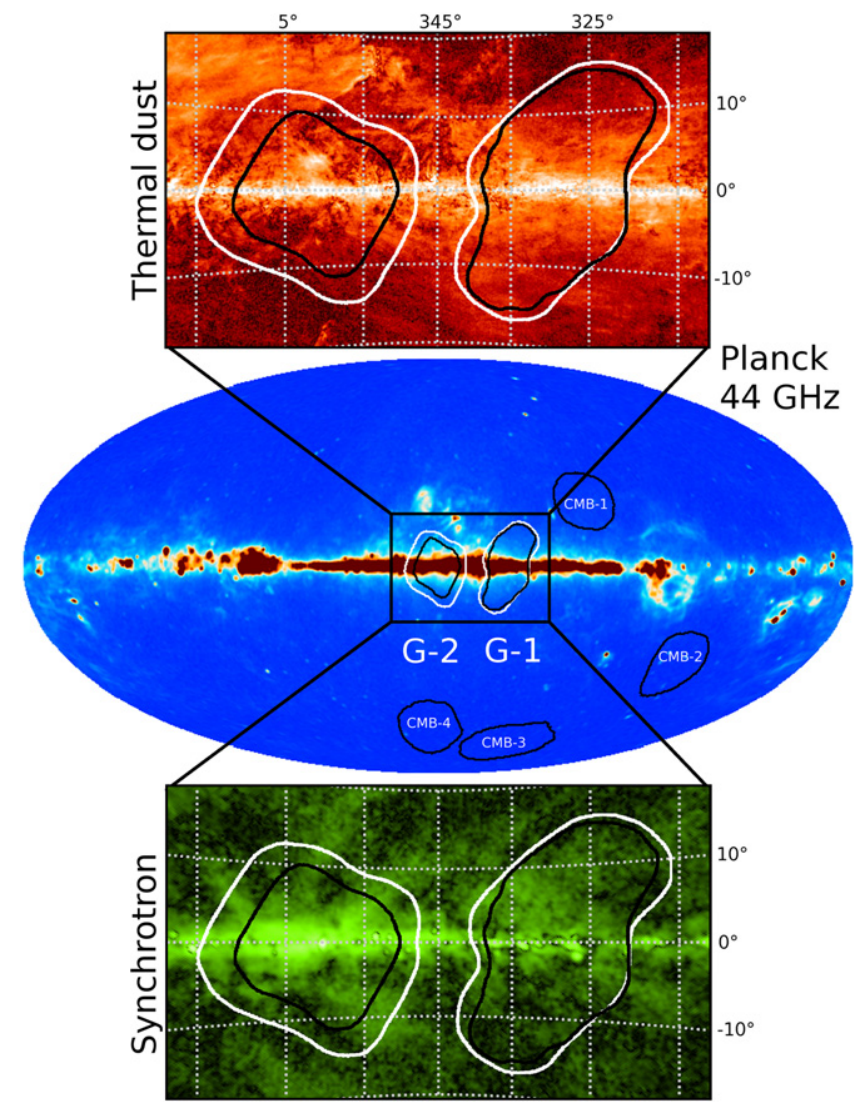

Figure 1. Positions of the QUIET fields superimposed on Planck foreground maps. The white and black outlines of the Galactic fields show the masks applied to the W-band $(95 \mathrm{GHz})$ and Q-band $(43 \mathrm{GHz})$ data, respectively. The central map is the Planck $44 \mathrm{GHz}$ temperature map, smoothed with a $1^{\circ}$ FWHM Gaussian beam, with an intensity range from -0.2 to $1 \mathrm{mK}$. The upper and lower panels compare the QUIET fields to the Planck polarized thermal dust and synchrotron foreground maps. Grid cell width is $10^{\circ}$.

addition with external data sets. The following analysis is based entirely on the maximum-likelihood pipeline.

\subsection{Observations and Data Selection}

The full, unfiltered data set consists of more than $10,000 \mathrm{hr}$ of observations taken from the Chajnantor plateau in Chile between 2008 October and 2010 December, covering two frequency bands $(\mathrm{Q}$ and $\mathrm{W}$, with center frequencies 43 and $95 \mathrm{GHz}$, respectively) and six separate fields on the sky, not counting various calibration targets. Four of these fields were selected as the primary low-foreground patches from which CMB constraints were derived (QUIET Collaboration et al. 2011, 2012a). To constrain polarized foregrounds at the same frequencies, two high-foreground fields targeting the Galactic plane were also included in the observation schedule, and these measurements are the subject of this paper. These fields were observed when no primary CMB targets were available, effectively filling in right ascension "gaps" in the observing schedule. Their positions on the sky are indicated in Figure 1, together with corresponding patches of the polarized synchrotron and thermal-dust maps recently published by Planck (Planck Collaboration X 2015). Adopting the notation introduced in QUIET Collaboration et al. (2011), we will refer to the two Galactic fields as G-1 and G-2. The line of sight in G-1 cuts through the Centaurus arm as well as the tangent region of the Norma spiral arm (García et al. 2014), while G-2 contains the Galactic center region. Thus, these fields cover the two most populated areas of the Galactic disk in terms of molecular gas (and therefore dust).

Summary characteristics are provided for both fields in Table 1, including positions, sky area, effective noise levels, and basic data-quality statistics. Regarding systematic uncertainties, we include only the absolute responsivity and polarization angle in Table 1 because these are the most important ones for foreground analysis. We discuss the level of temperature-to-polarization leakage in our maps in Section 3.1. For a complete discussion of systematic uncertainties relevant for B-mode analysis, we refer the interested reader to QUIET Collaboration et al. (2012b).

The basic observing block of the QUIET scanning strategy was a so-called constant-elevation scan (CES), in which the sky drifts through the field of view while the telescope performs a simple periodic azimuth slew of fixed amplitude. For the four CMB fields and the G-1 field, the scan width was $15^{\circ}$, while for G-2 it was $10^{\circ}$. Once the target field center drifted through the field of view by the same amount, the observing elevation was changed either to the opposite edge of the same field, thereby tracking the field on the sky in a set of discrete elevation steps, or to another field. The typical duration of a single CES was 30-60 minutes, depending on field size and elevation. In addition, the instrument was rotated about the optical axis (we refer to this as a deck rotation) in steps of $45^{\circ}$, typically once a week, for a total of eight angles. The combination of natural cross-linking from sky rotation and frequent deck rotations provided excellent modulation properties, suppressing many important instrumental systematic effects (QUIET Collaboration et al. 2012b).

As described in QUIET Collaboration et al. (2011, 2012a), the CMB-oriented QUIET data-reduction process was based on a fully blind analysis philosophy, in which all data cuts, filters, and main processing steps were defined and finalized before inspecting any final science results, including power spectra and cosmological parameters. In this process, each CES-diode (i.e., the CES time-stream from an individual detector diode) was scanned for signs of contamination and removed from the data set if any problematic issues were identified. The selection criteria assumed a low signal-to-noise ratio in any single CES, and relied strongly on accurate noise and $\chi^{2}$ modeling. This assumption, while valid for observations of the low-foreground CMB sky, does not hold for the Galactic patches considered in this paper. Rather, the amplitude of the Galactic polarization signal is sufficiently high that the strongest signals are visible even in a single CES, in particular at $43 \mathrm{GHz}$. Under the default CMB-targeted selection criteria, a large fraction of the Galactic observations would be automatically excluded for this reason alone, and the default pipeline is therefore not suitable for Galactic fields. On the other hand, the same high signal-tonoise ratio also implies that the fractional errors due to noisemodeling errors are much less important for Galactic than for $\mathrm{CMB}$ analysis.

For these reasons, we adopt the following simplified dataselection criteria in this paper. First, we apply the default selection pipeline to eliminate obviously void scans, but exclude any tests that depend directly on fits of noise quality. The CES removed in this step include those affected by faulty hardware, and those for which the moon was within the telescope's sidelobes. Second, we manually remove scans with 
Table 1

Summary and Comparison of Field Characteristics ${ }^{\mathrm{a}}$

\begin{tabular}{|c|c|c|c|c|}
\hline \multirow[b]{2}{*}{ Feature } & \multicolumn{2}{|c|}{ Q Band } & \multicolumn{2}{|c|}{ W Band } \\
\hline & G-1 & G-2 & G-1 & G-2 \\
\hline Field center, $(l, b)$ & $\left(329^{\circ}, 0^{\circ}\right)$ & $\left(0^{\circ}, 0^{\circ}\right)$ & $\left(329^{\circ}, 0^{\circ}\right)$ & $\left(0^{\circ}, 0^{\circ}\right)$ \\
\hline Sky area & $483 \mathrm{deg}^{2}$ & $301 \mathrm{deg}^{2}$ & $573 \mathrm{deg}^{2}$ & $539 \mathrm{deg}^{2}$ \\
\hline$N_{\text {pix }}\left(N_{\text {side }}=512\right)$ & 36,831 & 22,983 & 43,668 & 41,090 \\
\hline \multicolumn{5}{|l|}{ FWHM angular resolution } \\
\hline QUIET & \multicolumn{2}{|c|}{$27 ! 3$} & \multicolumn{2}{|c|}{$12 ! 8$} \\
\hline WMAP & \multicolumn{2}{|c|}{$30 ! 6$} & \multicolumn{2}{|c|}{$13 ! 2$} \\
\hline Planck & \multicolumn{2}{|c|}{$27 ! 0$} & \multicolumn{2}{|c|}{$\ldots$} \\
\hline \multicolumn{5}{|l|}{ Effective frequency, $\nu_{\text {eff }}$} \\
\hline QUIET & \multicolumn{2}{|c|}{$43.1 \mathrm{GHz}$} & \multicolumn{2}{|c|}{$94.5 \mathrm{GHz}$} \\
\hline WMAP & \multicolumn{2}{|c|}{$40.5 \mathrm{GHz}$} & \multicolumn{2}{|c|}{$94.2 \mathrm{GHz}$} \\
\hline Planck & \multicolumn{2}{|c|}{$44.1 \mathrm{GHz}$} & \multicolumn{2}{|c|}{$\ldots$} \\
\hline \multicolumn{5}{|l|}{ Noise $Q / U$ rms per $7^{\prime}$ pixel } \\
\hline QUIET & $17 \mu \mathrm{K}$ & $24 \mu \mathrm{K}$ & $15 \mu \mathrm{K}$ & $21 \mu \mathrm{K}$ \\
\hline WMAP & $58 \mu \mathrm{K}$ & $64 \mu \mathrm{K}$ & $96 \mu \mathrm{K}$ & $108 \mu \mathrm{K}$ \\
\hline Planck & $52 \mu \mathrm{K}$ & $55 \mu \mathrm{K}$ & $\ldots$ & $\ldots$ \\
\hline \multicolumn{5}{|c|}{ Linear regression $(y=a x+b)$ slope, $a$ (Section 3.2) } \\
\hline$x=$ QUIET $; y=W M A P, Q$ & $1.06 \pm 0.04$ & $1.05 \pm 0.04$ & $\ldots$ & $\ldots$ \\
\hline$x=$ QUIET $; y=W M A P, U$ & $1.11 \pm 0.17$ & $1.00 \pm 0.03$ & $\ldots$ & $\cdots$ \\
\hline$x=$ QUIET $; y=$ Planck, $Q$ & $1.33 \pm 0.30$ & $0.95 \pm 0.11$ & $\ldots$ & $\ldots$ \\
\hline$x=$ QUIET $; y=$ Planck,$U$ & $0.86 \pm 0.12$ & $1.00 \pm 0.04$ & $\ldots$ & $\ldots$ \\
\hline$x=W M A P ; y=$ Planck, $Q$ & $1.19 \pm 0.27$ & $0.90 \pm 0.14$ & $\ldots$ & $\ldots$ \\
\hline$x=W M A P ; y=$ Planck, $U$ & $0.78 \pm 0.14$ & $1.00 \pm 0.05$ & $\ldots$ & $\ldots$ \\
\hline \multicolumn{5}{|c|}{$\begin{array}{l}\text { Noise-weighted mean and standard deviation of deck split null map } \\
\text { (Section 3.1) }\end{array}$} \\
\hline Stokes $Q$ & $0.22 \pm 1.22 \mu \mathrm{K}$ & $-0.32 \pm 1.08 \mu \mathrm{K}$ & $0.09 \pm 1.03 \mu \mathrm{K}$ & $-0.17 \pm 1.01 \mu \mathrm{K}$ \\
\hline Stokes $U$ & $-0.03 \pm 1.16 \mu \mathrm{K}$ & $0.10 \pm 1.05 \mu \mathrm{K}$ & $-0.12 \pm 1.04 \mu \mathrm{K}$ & $0.20 \pm 1.01 \mu \mathrm{K}$ \\
\hline \multicolumn{5}{|c|}{ QUIET systematic uncertainties (QUIET Collaboration et al. 2012b) } \\
\hline Absolute responsivity & \multicolumn{2}{|c|}{$6 \%$} & \multicolumn{2}{|c|}{$8 \%$} \\
\hline Absolute detector angle. & \multicolumn{2}{|c|}{$1: 7$} & \multicolumn{2}{|c|}{0.5} \\
\hline
\end{tabular}

Note.

${ }^{\mathrm{a}}$ Note that Planck has not yet released W-band polarization maps, and the corresponding table entries are therefore empty.

poor pointing (i.e., scans that did not hit the main target field) or short duration. Likewise, we remove a small, discrete set of scans that on visual inspection was found to exhibit largeamplitude striping aligned with the scanning strategy. The cause of this latter effect is unknown. It is illustrated in Figure 2 through two single-CES, single-detector-module maps from the W-band observations of field G-1. The left-hand panel shows a normal map, while the right-hand panel shows a contaminated map. The data containing the highest proportion of these CES are the W-band observations of field G-1, in which they make up $10 \%$ of the full data set. Although a substantial fraction of these could be saved through more aggressive filtering, considering the high signal-to-noise ratio of these Galactic sky maps, we prefer to minimize systematic effects rather than instrumental noise, and conservatively remove all CES that contain at least one striped single-detector map.

Table 2 summarizes the data-selection statistics, both in terms of individual cuts and total observing efficiency. In total, 392 (868) observation hours are included in the final Q-band (W-band) maps, corresponding to an acceptance rate of about $90 \%$, depending on field and frequency. For comparison, the acceptance rate obtained in the CMB-oriented QUIET analyses was 70\%-73\% (QUIET Collaboration et al. 2011, 2012a).
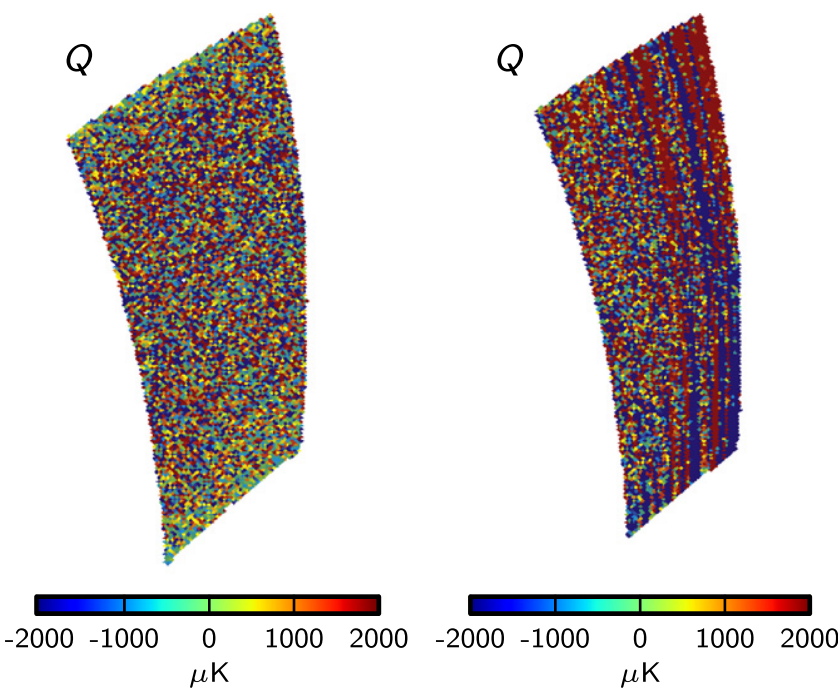

Figure 2. Example of scan cut due to excessive striping. Both maps show the data taken by a single detector module during a single CES. The left-hand map shows a normal CES (CES id no. 1808), while the right-hand map exhibits striping aligned with the scanning strategy (CES id no. 1826). All CES containing at least one such feature were cut from the analysis. Both example CES are taken from the W-band (95 GHz) observations of field G-1. 
Table 2

Summary of Data Selection ${ }^{\mathrm{a}}$

\begin{tabular}{lccccc}
\hline \hline & \multicolumn{2}{c}{ Q Band } & & \multicolumn{2}{c}{ W Band } \\
\cline { 2 - 3 } \cline { 5 - 6 } Description & G-1 & G-2 & & G-1 & G-2 \\
\hline Total CES count & 295 & 189 & & 568 & 352 \\
Automatic cuts & 1 & 13 & & 27 & 8 \\
Poor pointing & 8 & 0 & & 4 & 4 \\
Short duration & 3 & 0 & & 14 & 0 \\
Excessive striping & 5 & 2 & & 62 & 27 \\
CES count after cuts & 278 & 174 & & 461 & 313 \\
Observing time & $303 \mathrm{hr}$ & $92 \mathrm{hr}$ & & $595 \mathrm{hr}$ & $273 \mathrm{hr}$ \\
Efficiency & $94 \%$ & $91 \%$ & & $83 \%$ & $90 \%$ \\
\hline
\end{tabular}

Note.

${ }^{a}$ List of the number of CES cut from the final data set by the cut criteria described in Section 2.1.

\subsection{Mapmaking}

Given a set of time-ordered data, we employ full maximumlikelihood mapmaking to estimate unbiased sky maps, $\boldsymbol{m}$, by solving the normal equations (e.g., Tegmark 1997; Keskitalo et al. 2010)

$$
\boldsymbol{m}=\left(\boldsymbol{P}^{T} \tilde{\boldsymbol{N}}^{-1} \tilde{\boldsymbol{F}} \boldsymbol{P}\right)^{-1} \boldsymbol{P}^{T} \tilde{\boldsymbol{N}}^{-1} \tilde{\boldsymbol{F}} \tilde{\boldsymbol{d}}
$$

Here symbols marked by $\sim$ denote pure time-domain objects, while $\boldsymbol{P}$ and $\boldsymbol{m}$ denote (at least partially) map-domain objects. Specifically, $\boldsymbol{P}$ represents the pointing matrix, as defined by the default QUIET pointing model (QUIET Collaboration et al. $2012 \mathrm{a}) ; \tilde{N}$ is the time-domain noise covariance matrix, given by a $1 / f$ noise model; $\tilde{\boldsymbol{F}}$ represents a general time-domain filter; and $\tilde{\boldsymbol{d}}$ denotes the actual time-ordered data. The corresponding map-domain noise covariance matrix is

$$
\boldsymbol{N}=\left(\boldsymbol{P}^{T} \tilde{\boldsymbol{N}}^{-1} \tilde{\boldsymbol{F}} \boldsymbol{P}\right)^{-1}\left(\boldsymbol{P}^{T} \tilde{\boldsymbol{F}}^{T} \tilde{\boldsymbol{N}}^{-1} \tilde{\boldsymbol{F}} \boldsymbol{P}\right)\left(\boldsymbol{P}^{T} \tilde{\boldsymbol{N}}^{-1} \tilde{\boldsymbol{F}} \boldsymbol{P}\right)^{-1}
$$

Conversion between measured voltages and thermodynamic temperature units, bandpass integration, and polarization-angle definitions are all implicitly described by the pointing matrix, $\boldsymbol{P}$. For full details and conventions, see QUIET Collaboration et al. (2011, 2012a, 2012b).

We use a HEALPix ${ }^{25} N_{\text {side }}=512$ grid with $7^{\prime} \times 7^{\prime}$ pixels for our maps, sufficient to support the 12!.8 FWHM angular resolution of the QUIET W-band channel. The QUIET Q-band channel has angular resolution of 27!3 FWHM, and could in principle be pixelized with $14^{\prime} \times 14^{\prime}$ pixels; however, for consistency we pixelize both channels with the same grid. The $W M A P$ polarization sky maps also use $N_{\text {side }}=512$ grids.

The total number of observed Q-band (W-band) pixels is 47,288 (52,391) and 45,162 (56,216) for G-1 and G-2, respectively. However, many of these pixels are observed only a few times, and therefore have low signal-to-noise ratio. In order to reduce the cost of subsequent matrix operations and data volumes, and acknowledging the fact that we later will coadd our maps with WMAP maps, we apply a mask to each full map, removing any pixels for which the effective QUIET noise rms is more than 1.5 times the corresponding WMAP noise rms. The resulting masks are shown as black and white contours in Figure 1 for both $\mathrm{Q}$ and $\mathrm{W}$ bands.

\footnotetext{
${ }^{25}$ http://healpix.sourceforge.org
}

While the QUIET CMB analysis used several complementary time-domain filters, the requirements for foreground observations are somewhat different (see above). For these new maps, we have found that a mildly apodized high-pass filter with a cut-off frequency of $0.5 f_{\text {scan }}$ is sufficient to produce maps with no obvious artifacts, where $f_{\text {scan }} \approx 0.1 \mathrm{~Hz}$ is the scanning frequency of the telescope. The only exception is a specific large-scale feature in the G-1 field due to poor crosslinking. However, as described below, rather than imposing a stronger time-domain filter in this case, we project out all harmonic modes with $\ell \leqslant 10$ in the pixel domain, to avoid excessive anisotropic filtering. No low-pass filters are applied, in view of the fact that Galactic features tend to be strongly localized and full angular resolution is particularly important.

\subsection{Co-addition with External Data Sets}

QUIET is for all practical purposes insensitive to physical modes with wavelengths comparable to the size of the observed field. The QUIET field diameter of $\lambda \sim 20^{\circ}$ thus suggests a loss of sensitivity for modes of $\ell \lesssim 18 .^{26}$ Although not vital for CMB power spectrum estimation, these modes are important for astrophysical foreground inference. We therefore co-add QUIET with an external large-scale experiment in order to produce optimal all-scale maps. Algorithmically, the coaddition is given by an inverse-variance-weighted sum of the form

$$
\boldsymbol{m}_{\mathrm{tot}}=\left(\sum_{i} \boldsymbol{N}_{i}^{-1}\right)^{-1}\left(\sum_{i} \boldsymbol{N}_{i}^{-1} \boldsymbol{m}_{i}\right),
$$

where the sums run over experiments, and $\boldsymbol{N}_{i}$ represents the noise covariance matrix for the $i$ 'th experiment. The covariance matrix of the final map is

$$
\boldsymbol{N}_{\mathrm{tot}}=\left(\sum_{i} \boldsymbol{N}_{i}^{-1}\right)^{-1} \text {. }
$$

If a set of $N$ modes (which may be organized column-wise into an $N_{\text {pix }} \times N$ matrix $\boldsymbol{V}$ ) happens to be affected by instrumental systematic errors in a given experiment, the $N$ modes can be projected out from the corresponding experiment covariance matrix before co-addition. This is most easily done by means of the Sherman-Morrison-Woodbury formula (e.g., Woodbury 1950),

$$
\boldsymbol{N}_{i} \rightarrow \boldsymbol{N}_{i}-\boldsymbol{N}_{i} \boldsymbol{V}\left(\boldsymbol{V}^{T} \boldsymbol{N}_{i}^{-1} \boldsymbol{V}\right)^{-1} \boldsymbol{V}^{T} \boldsymbol{N}_{i}
$$

Effectively, this operation assigns infinite variance to all modes in $\boldsymbol{V}$, ensuring that those modes do not contribute to the final map. In practice, we will use this operation to project out the largest-scale modes to which we can be certain that QUIET, due to its finite field size, has no sensitivity, by letting $\boldsymbol{V}$ consist of all spherical harmonics with $\ell \leqslant 10$.

We use the notation described above to define an instrumentspecific weight operator, $\boldsymbol{F}_{i}$, of the form

$$
\boldsymbol{F}_{i}=\left(\sum_{j} \boldsymbol{N}_{j}^{-1}\right)^{-1} \boldsymbol{N}_{i}^{-1}
$$

\footnotetext{
${ }^{26}$ In the QUIET CMB analyses, a lower limit of $\ell=25$ was chosen for CMB power spectrum estimation from QUIET data (QUIET Collaboration et al. 2011, 2012a).
} 
which simply measures the relative weight carried by experiment $i$ of each mode in the final map. For instance, $\boldsymbol{F}_{\mathrm{Q}} \boldsymbol{m}_{\mathrm{Q}}$ is the contribution from QUIET to the total map, $\boldsymbol{m}_{\text {tot }}$. Note that the sum over these operators is unity, $\sum_{i} \boldsymbol{F}_{i}=1$, ensuring that the final map will be unbiased irrespective of instrument-specific filtering, as long as each individual map is inherently unbiased.

Although QUIET, WMAP, and Planck all nominally observe at $\mathrm{Q}$ band, they do have slightly different bandpasses and effective frequencies, as listed in Table 1. To account for these differences, we rescale the WMAP and Planck maps to the nominal QUIET frequency $\nu_{\mathrm{Q}}$ before co-addition, assuming a synchrotron-type power-law index across the bands. Explicitly, the scaling factor for converting a map from frequency $\nu_{i}^{\text {eff }}$ to $\nu_{\mathrm{Q}}^{\text {eff }}$ is

$$
\gamma_{i}=\frac{g\left(\nu_{\mathrm{Q}}^{\text {eff }}\right)}{g\left(\nu_{i}^{\text {eff }}\right)}\left(\frac{\nu_{\mathrm{Q}}^{\text {eff }}}{\nu_{i}^{\text {eff }}}\right)^{\beta},
$$

where

$$
g(\nu)=\frac{\left(e^{x}-1\right)^{2}}{x^{2} e^{x}}, \quad x=\frac{h \nu}{k_{\mathrm{B}} T_{\mathrm{CMB}}}
$$

is the conversion factor between brightness and differential thermodynamic temperature. Here, $h$ and $k_{\mathrm{B}}$ denote the Planck and Boltzmann constants, and $T_{\mathrm{CMB}}=2.7255 \mathrm{~K}$ is the $\mathrm{CMB}$ monopole temperature. In order to avoid circularity in the analysis process, we adopt the synchrotron spectral index values reported by Fuskeland et al. (2014) for these re-scaling factors, not those that will be derived from the QUIET maps themselves in Section 4. Specifically, Fuskeland et al. partitioned the whole sky (excluding bright compact objects and a region around the Galactic center of radius $1^{\circ}$ ) into 24 regions, and estimated the synchrotron spectral index for each region from the WMAP $\mathrm{K}$ - and Ka-band polarization sky maps. For G-1, we adopt the mean of their regions 23 and 24 (see Figure 1 in Fuskeland et al. 2014), resulting in $\beta_{\mathrm{G}-1}=-2.93 \pm 0.01$, while for G-2 we use the mean of regions 15 and 24, resulting in $\beta_{\mathrm{G}-2}=-3.00 \pm 0.009$. For WMAP, these spectral indices translate into scaling factors for G-1 and G-2 of 0.84 and 0.83 , respectively. For Planck the corresponding factors are 1.067 and 1.069. If we instead were to adopt the spectral indices derived in Section 4 from QUIET (i.e., $\beta=-3.12 \pm 0.06$ ), these numbers would change by $1.2 \%$ and $0.7 \%$ for WMAP, and by $0.4 \%$ and $0.3 \%$ for Planck. The impact of the precise value of the assumed spectral index is small compared to the intrinsic absolute responsivity uncertainty of $6 \%$ in the QUIET observations (QUIET Collaboration et al. 2011). For $\mathrm{W}$ band, the difference between the WMAP and QUIET frequencies is negligible, and we omit any re-scaling in this case. A Planck W-band polarization map is not yet available (Planck Collaboration I 2015).

\subsection{Detailed Analysis of Q-band G-1 Field}

Before presenting the results from our analysis, it is useful to gain some intuition regarding the co-addition process described above. We therefore present the combination of the QUIET and
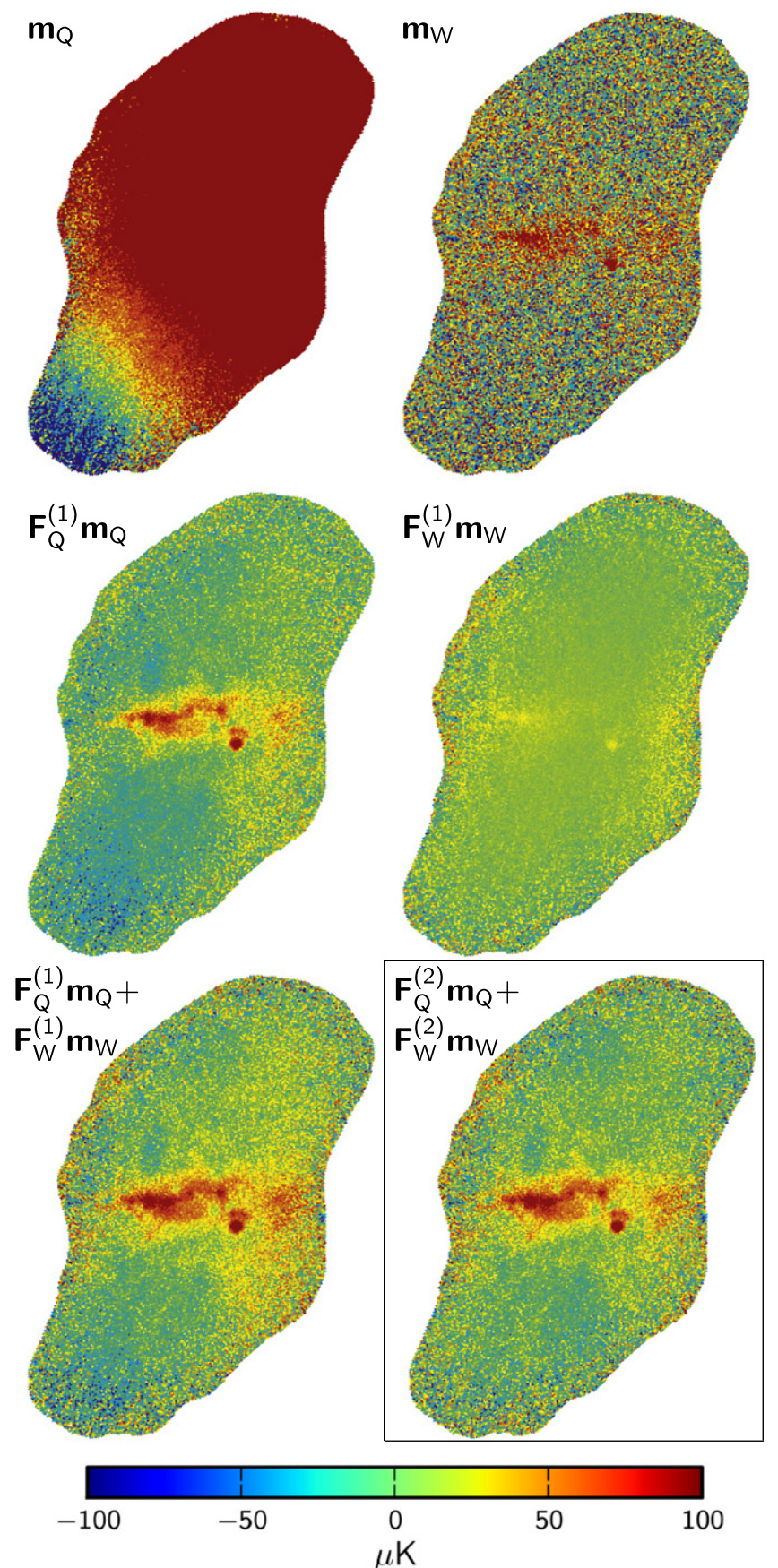

Figure 3. Example of map co-addition, applied to the Q-band QUIET and WMAP Stokes $Q$ maps of the G-1 field. The top row shows the QUIET maximum-likelihood map, $\boldsymbol{m}_{\mathrm{Q}}$, and the WMAP 9-year map, $\boldsymbol{m}_{\mathrm{W}}$. The middle row shows the contribution to the co-added map from each data set, computed by applying the corresponding weight operators, defined in Section 2.3, to each map: $\boldsymbol{F}_{\mathrm{Q}}^{(1)} \boldsymbol{m}_{\mathrm{Q}}$ and $\boldsymbol{F}_{\mathrm{W}}^{(1)} \boldsymbol{m}_{\mathrm{W}}$. The co-added map, shown in the bottom left panel, is the sum of the two contributions. The framed panel (bottom right) shows an equivalent co-added map made using a version of the QUIET map where all modes of $\ell \leqslant 10$ have been discarded prior to co-addition; see Section 2.4 for further details.

WMAP Q-band G-1 maps in Figure 3. The top row shows the raw maps from each experiment separately. The QUIET map is dominated by an essentially unconstrained mode with a gradient extending from the upper right to lower left. Qualitatively similar features appear in all QUIET maximumlikelihood maps, but with an amplitude that varies strongly 
from field to field. In terms of how well the largest scales are constrained, the G-1 field is by far the worst of all six QUIET fields, whereas G-2 is one of the best. The fundamental difference between these two extreme cases lies in their degree of cross-linking coupled to the size and shape of the field. G-1 is neither a primary CMB field nor a particularly useful calibration source. As a result, it was observed through a relatively small set of scanning directions, from the upper right to the lower left edges in Figure 3. Moreover, only half of the amplitude was scanned within a single CES, resulting in two only partially overlapping and almost independent CES sets. Consequently, large-scale modes aligned with this direction are poorly constrained.

The G-2 field, on the other hand, covers the Galactic center, including the Galactic center arc, the strongest polarized object within the QUIET patches, and therefore is a particularly useful calibration source, both for absolute responsivity and for pointing reconstruction (QUIET Collaboration et al. 2012b). As a result, this field was observed from many different angles, leading to a more symmetric map. Additionally, the G-2 field was smaller to reduce the noise per sky area and could be scanned from edge to edge within a single CES. Thus, even the large-scale modes are quite well constrained in G-2, and the raw G-2 map shows only weak evidence for spurious largescale gradients.

Substantial benefits can be obtained by co-adding data from QUIET with some large-scale experiments such as WMAP and/ or Planck. The middle row of Figure 3 shows the inversevariance-weighted component maps for QUIET and WMAP, $\boldsymbol{F}_{\mathrm{Q}} \boldsymbol{m}_{\mathrm{Q}}$ and $\boldsymbol{F}_{\mathrm{W}} \boldsymbol{m}_{\mathrm{W}}$, as estimated from Equation (6). QUIET dominates the small-scale features in terms of the signal-tonoise ratio, while WMAP dominates the large-scale modes. The previously dominating gradient in the raw QUIET map is effectively suppressed, and the weighted sum of the two contributions is shown in the bottom left panel.

Nevertheless, a non-zero gradient is present even in the coadded map, albeit at a greatly reduced level. Neither WMAP nor Planck observe this mode; it is clearly due to an instrumental effect in QUIET, perhaps ground pick-up (QUIET Collaboration et al. 2012b). Irrespective of its origin, but confident it is an artifact in the QUIET observations, we apply an additional real-space filter that projects out all harmonic modes with $\ell \leqslant 10$ from the QUIET map, as described by Equation (5). We therefore adopt these few modes entirely from WMAP, rather than estimating them in terms of a weighted mean of the two experiments. The result is shown in the lower right panel of Figure 3, and this map appears astrophysically more reasonable than the unfiltered version in the lower left panel. We evaluate the need for this filtering by comparing the rms of the QUIET maps before and after applying it. In $Q$ band, filtering decreases the rms of the G-1 maps by more than $50 \%$, whereas the corresponding value for field G-2 is a mere $2 \%$. Similar results are found for the W-band maps. Hence we conclude that such filtering is prudent in the case of field G-1, but unneccessary for the far better constrained case of field G-2. All co-added maps for G-1 presented in the following have been derived using QUIET maps pre-filtered in this way.

\section{SKY MAPS}

We are now ready to present the Galactic field sky maps as observed by QUIET. In order to avoid excessive repetition, we will focus our discussion around the Q-band G-2 field, i.e., around the Galactic center region at $43 \mathrm{GHz}$. Corresponding plots and discussions for the remaining three fields (G-1 at $\mathrm{Q}$ band, and both G-1 and G-2 at $\mathrm{W}$ band) are given in the Appendix.

\subsection{QUIET-only Maps}

The third row of Figure 4 shows the inverse-variance-filtered QUIET G-2 maps, $\boldsymbol{F}_{\mathrm{Q}} \boldsymbol{m}_{\mathrm{Q}}$. As described in the next section, we choose for now to include QUIET and WMAP, but not Planck, in the weighted sum defined by Equations (3)-(6). Thus, the modes that are weighted down by the $\boldsymbol{F}_{\mathrm{Q}}$ operator are those for which WMAP has lower instrumental noise than QUIET, as measured by the respective noise-covariance matrices. This translates to the large-scale modes, as illustrated in Section 2.4.

The bottom row shows corresponding (half-difference) nullmaps. These are derived by first dividing the full time-ordered QUIET data set according to the angle of deck rotation, with one set consisting of data taken with deck angles $0^{\circ}, 90^{\circ}, 180^{\circ}$, and $270^{\circ}$, and the other with deck angles $45^{\circ}, 135^{\circ}, 225^{\circ}$, and $315^{\circ}$. Independent maps are made from each subset, which are then subtracted. In the absence of systematic errors, any such null-map should contain instrumental noise only, and, as already mentioned, the QUIET analysis is fundamentally dependent on understanding null-maps. In the original CMBoriented analysis, more than 20 different data splits were included. In this paper, we focus on the deck-angle split alone, because it is the most stringent test for Galactic fields. Noiseweighted mean and standard deviation values of the deck-split null-maps of all four fields, given in Table 1, show that these maps are consistent with the expected Gaussian distribution. The only significant excesses in the Q-band G-2 null-map are two small-scale features, one toward the very Galactic center, the other toward a compact object at Galactic coordinates $(l, b)=(353: 17,0.76)$ that is identified as PCCS1 030 G353.17+00.76 in the Planck Catalog of Compact Sources (Planck Collaboration VII 2011).

The most likely explanation for these excesses is so-called temperature-to-polarization ( $I$-to- $Q / U$ ) leakage. Each QUIET MMIC module contains four detector diodes, two measuring $Q$, two measuring $U$, as defined by the local detector coordinate system (QUIET Collaboration et al. 2012b). Based on sky-dips (i.e., elevation nods designed to monitor relative gain variations) and lunar and Galactic observations, QUIET Collaboration et al. (2011) found that the instantaneous temperature-to-polarization leakage for the Q-band detectors was about $1 \%$ in $Q$ and $0.2 \%$ in $U$. Modulation by both sky and deck rotations effectively suppresses this effect in final maps. The deck-angle null-test shown in Figure 4 therefore provides a very strict upper limit on the net final leakage.

To quantify this effect more accurately, we compare the nullmap polarization amplitude with the WMAP Q-band temperature map in Figure 5, both smoothed to an effective resolution of $40^{\prime}$ FWHM to reduce noise. Comparing the two maps visually, the qualitative correlation between the polarization excess and the temperature signal is obvious. Furthermore, we find that the peak value of the polarization amplitude in the null 

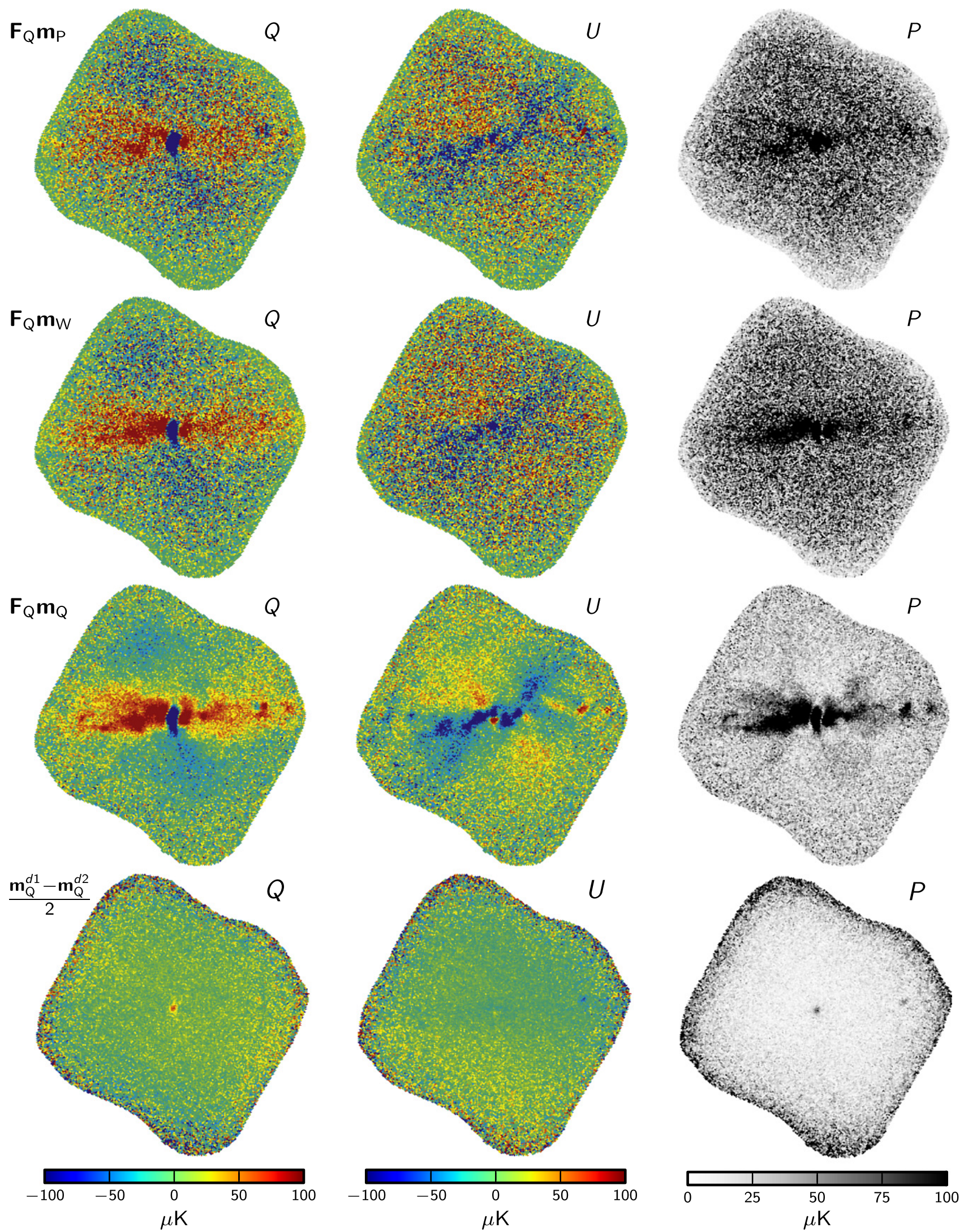

Figure 4. Inverse-noise-variance-weighted Q-band (43 GHz) maps of the G-2 field (centered at Galactic coordinates $(l, b)=\left(0^{\circ}, 0^{\circ}\right)$ ), for Planck, WMAP, and QUIET. Columns show, from left to right, Stokes $Q$, Stokes $U$, and polarization amplitude $P=\sqrt{Q^{2}+U^{2}}$. The top row shows the Planck map $\boldsymbol{m}_{\mathrm{P}}$, filtered to only contain the small-scale modes observable by QUIET, as determined by the QUIET weighting operator $\boldsymbol{F}_{\mathrm{Q}}$ defined in Section 2.3. Rows 2 and 3 show the equivalent maps for WMAP and QUIET, respectively. The bottom row shows the half-difference null maps of the deck-split QUIET data; see Section 3.1 for full details. 

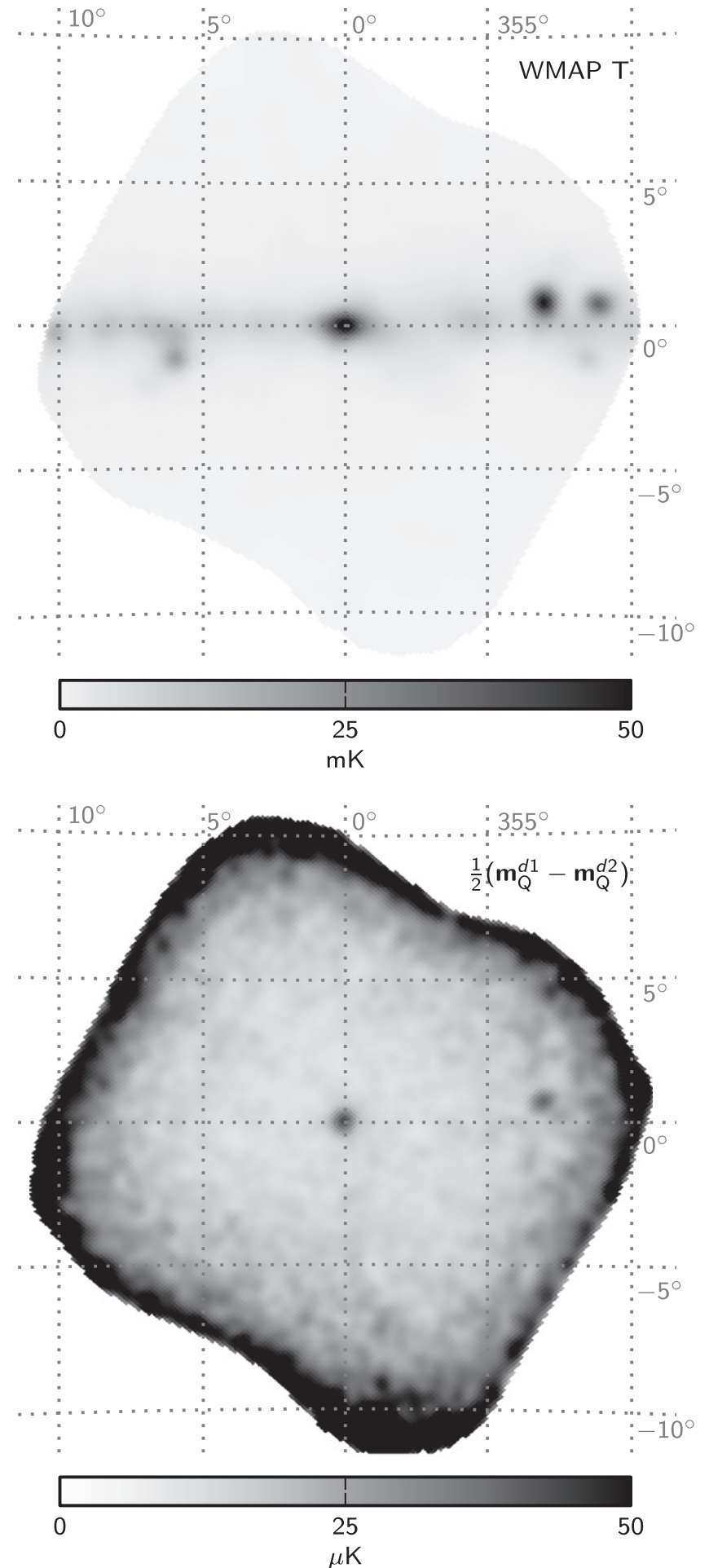

Figure 5. $I$-to- $Q / U$ leakage in the QUIET Q-band ( $43 \mathrm{GHz}$ ) G-2 field (centered on Galactic coordinates $\left.(l, b)=\left(0^{\circ}, 0^{\circ}\right)\right)$. The top panel shows the WMAP9 Q-band temperature data. The bottom panel shows the half-difference map $\left(\boldsymbol{m}_{\mathrm{Q}}^{d 1}-\boldsymbol{m}_{\mathrm{Q}}^{d 2}\right) / 2$ of QUIET data, split by deck-angle (rotation angle of optical axis), in polarization amplitude $P=\sqrt{Q^{2}+U^{2}}$. Both maps have been smoothed to $40^{\prime}$ FWHM. Grid cell width is $5^{\circ}$.

map at the Galactic center is about $40 \mu \mathrm{K}$, while the corresponding peak temperature amplitude is $60 \mathrm{mK}$. Thus, the net $I$-to- $Q / U$ leakage is about $0.07 \%$ in the deck-split null map. In terms of total net polarization amplitude, this deck-split leakage corresponds to less than $4 \%$ of the full polarization signal of the Galactic center source. Again, after averaging over all possible polarization detector angles, these numbers will be significantly lower in the final maps.

\subsection{Comparison with Planck and WMAP}

The top two rows in Figure 4 show the Planck and WMAP maps, scaled to the QUIET frequency as per Equation (7), and filtered with the QUIET weight operator, i.e., $\boldsymbol{F}_{\mathrm{Q}} \boldsymbol{m}_{\mathrm{P}}$ and $\boldsymbol{F}_{\mathrm{Q}} \boldsymbol{m}_{\mathrm{W}}$. By removing the same large-scale basis functions from each map, all three can be directly compared without confusion from poorly constrained large-scale modes. A quantitative comparison between the filtered Q-band QUIET, WMAP, and Planck maps is given in Table 1 in the form of best-fit linear regression slopes (Petrolini 2014); corresponding W-band results are not provided, due to the very low signal-to-noise ratio of the WMAP W-band sky map and non-availability of the Planck $100 \mathrm{GHz}$ map.

Several interesting features may be seen in these maps. First and foremost, it is reassuring that all three experiments observe the same broad structures, namely the positive Galactic plane and bright negative Galactic center in Stokes $Q$, and the negative "wings" in Stokes $U$. However, there are noticeable differences as well, the most important of which is the much lower noise of the QUIET maps. While only broad features may be identified in the Planck and WMAP maps, even beamsized features may be picked out by eye in the QUIET map.

A second important but more subtle difference is the apparent amplitude of the Galactic plane in Stokes $Q$. Both QUIET and WMAP appear to be slightly brighter than Planck in the Galactic plane. This effect is visually more striking in Figure 6, where we show pairwise difference maps between all three experiments, all repixelized on a $55^{\prime} \times 55^{\prime}$ grid $\left(N_{\text {side }}=64\right)$ in order to suppress instrumental noise. From top to bottom, the three rows show QUIET-WMAP, QUIET-Planck, and WMAP-Planck. While QUIET and WMAP are consistent, Planck clearly shows a deficit in the Galactic plane compared to the other two experiments.

Another illustration of the same effect is provided in Figure 7, which shows the Stokes $Q$ differences with respect to QUIET of Planck (blue curves) and WMAP (red curves) as a function of Galactic longitude, averaged over a $|b| \leqslant 1.5$ latitude band around the Galactic plane. This corresponds to the three center-most rows of pixels in Figure 6, although the evaluation was in fact performed on the full-resolution maps. For comparison, we have also plotted the corresponding mean of the WMAP Q-band temperature map as dotted lines, but scaled with a factor of 0.002 . This signal would be produced by temperature-to-polarization leakage of $0.2 \%$. The thin colored lines correspond to multiplying the QUIET map by $\pm 6 \%$, which is the QUIET $1 \sigma$ uncertainty in absolute calibration (QUIET Collaboration et al. 2011). Finally, we have marked the Galactic center region within $1^{\circ}$ (i.e., the bright blue object in the Galactic center seen in Figure 4) with a gray band. As noted in Section 2, we assume a synchrotron spectral index of $\beta_{\mathrm{G}-2}=-3.00$ for this field, as estimated by Fuskeland et al. (2014). However, the bright center object is not included in this estimate, as its properties appear qualitatively different from the surrounding environment. In addition, the amplitude of this object is very large, reaching $2600 \mu \mathrm{K}$ at $30^{\prime}$ scales, and any difference maps like those considered here are therefore very sensitive to spectral index uncertainties. For example, if the spectral index is -2.7 rather than -3.0 , the effective difference between QUIET and WMAP would be $-40 \mu \mathrm{K}$, similar to what is seen in Figure 7. For now, we therefore exclude the central 

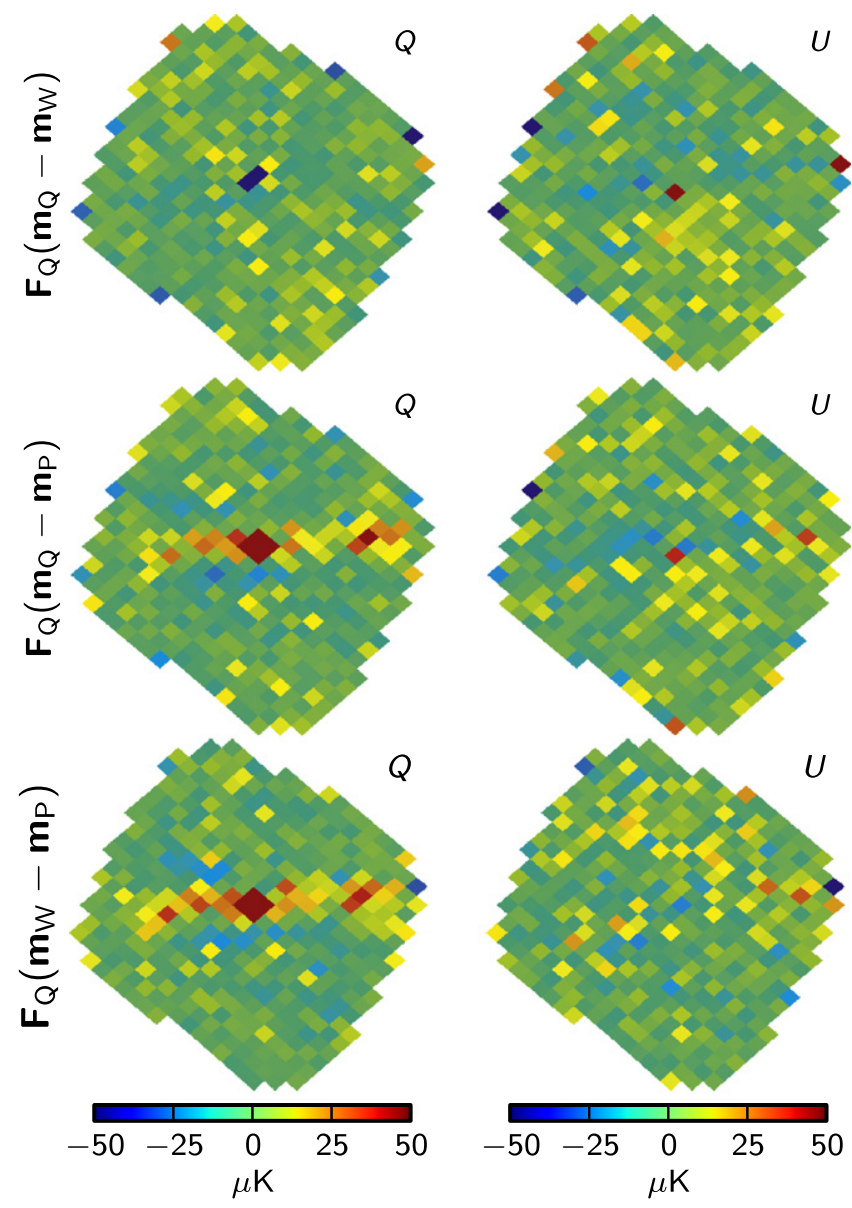

Figure 6. Pairwise differences of Q-band (43 GHz) maps of the G-2 (Galactic center) field, downgraded to HEALPix $N_{\text {side }}=64$ to suppress noise. All maps are weighted using the QUIET weight operator $\boldsymbol{F}_{\mathrm{Q}}$, defined in Section 2.3, retaining only the small-scale modes observed by QUIET in the differenced maps. Rows show, from top to bottom, QUIET-WMAP, QUIET-Planck, and $W M A P-P l a n c k$. Columns show Stokes $Q$ and Stokes $U$.

$|l| \leqslant 1^{\circ}$ region from our discussion, noting that further spectral index estimation work is required before conclusions can be drawn for this object.

Focusing on the remaining longitude region with $|l|>1^{\circ}$ in Figure 7, we see again the good agreement between QUIET and WMAP. The thick red line fluctuates around zero with an overall mean and standard deviation of $-1 \pm 3 \mu \mathrm{K}$. In Section 3.1, we derived an upper limit on the polarization amplitude uncertainty from temperature-to-polarization leakage of $\sim 4 \%$ in the QUIET deck-angle null-map, which is further suppressed in the full map due to cross-linking. The uncertainty in the QUIET maps due to such leakage is therefore well within the level indicated by the $\pm 6 \%$ uncertainty shown in the plot. In sum, we do not find any evidence for significant residual temperature-to-polarization leakage in the full signal maps, either in QUIET or WMAP.

For Planck, we see a systematic positive excess, consistent with Figure 6. In principle, this excess could be due to several different effects. However, its magnitude and spatial pattern suggest temperature-to-polarization leakage, as discussed extensively in Planck Collaboration II (2015). Compare the QUIET-Planck residual to the upper dotted line, which indicates the mean WMAP Q-band temperature signal as a function of longitude, scaled by a factor of 0.002 . Note, in

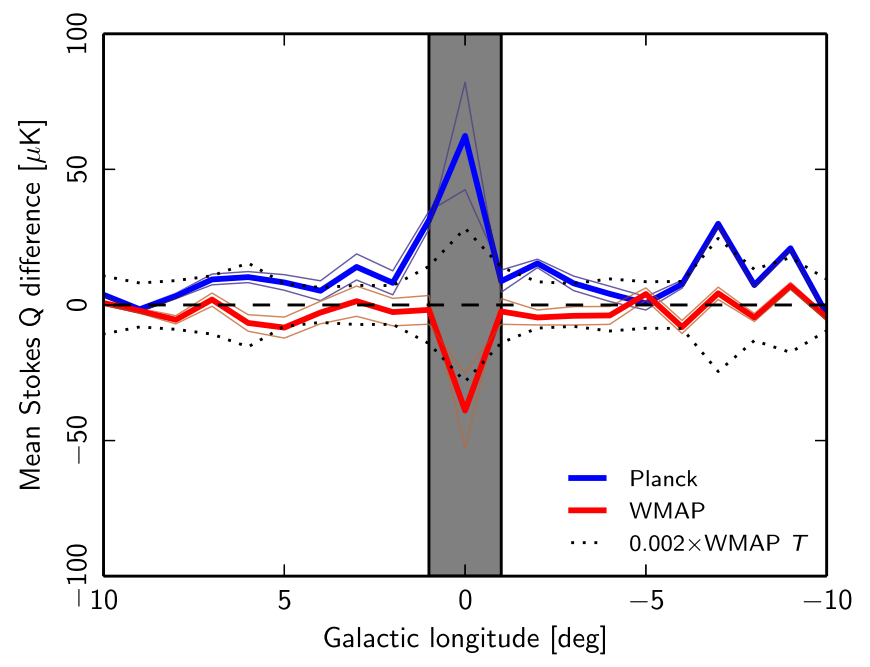

Figure 7. Latitude-averaged difference between QUIET and WMAP (red) and between QUIET and Planck (blue) Q-band (43 GHz) maps of field G-2 (Galactic center), in Stokes $Q$, evaluated over a latitude band around the Galactic plane of $|b| \leqslant 1$.5. All maps are filtered with the QUIET weight operator $\boldsymbol{F}_{\mathrm{Q}}$, defined in Section 2.3, retaining only the small-scale modes that are well constrained by QUIET. The colored regions indicate the absolute QUIET calibration uncertainty of $\pm 6 \%$. The dashed lines show the latitudeband-averaged WMAP Q-band temperature amplitude scaled by a factor of 0.002 , providing a rough template of $0.2 \%$ temperature-to-polarization leakage. The gray region marks an area in longitude $\pm 1^{\circ}$ around the Galactic center within which all results are dominated by uncertainties in the foreground spectral index.

particular, the correlated structures between longitudes $l \approx-5^{\circ}$ and $-10^{\circ}$. The dotted line provides an approximate template of the temperature-to-leakage signal. However, this template is only approximate, since the detailed leakage pattern will additionally depend on the Planck scanning strategy and detector orientation, of which the effects are not accounted for here. Note, however, that such a scanning strategy modulation can only reduce the correlation between the observed residual and the simple leakage model, and can never enhance it. Additionally, these features cannot be due to intrinsic spectral index variations (unlike the Galactic center), because WMAP agrees very well with QUIET in this region despite having a longer relative frequency lever arm than Planck.

Residuals at this level are consistent with the uncertainties for temperature-to-polarization leakage given in Planck Collaboration II (2015). Planck Collaboration X (2015) gives explicit leakage corrections based on detailed astrophysical foreground modeling; however, those models are necessarily associated with significant uncertainties because they depend sensitively on both instrument and foreground models, and, in particular, on the assumed bandpass properties of the instrument. The Planck $44 \mathrm{GHz}$ polarization map fails a few null-tests (Planck Collaboration I 2015), and is therefore not used in the Planck 2015 CMB likelihood (Planck Collaboration XI 2015). Thus, the new high-sensitivity QUIET maps presented in this paper represent a unique opportunity to improve the Planck leakage model in future analyses, by virtue of providing a clean and direct reference in the region of the sky with the highest signal-to-noise ratio.

\subsection{Co-added Sky Maps}

Given the qualitative differences between the Planck and QUIET maps reported above, we co-add the QUIET and 

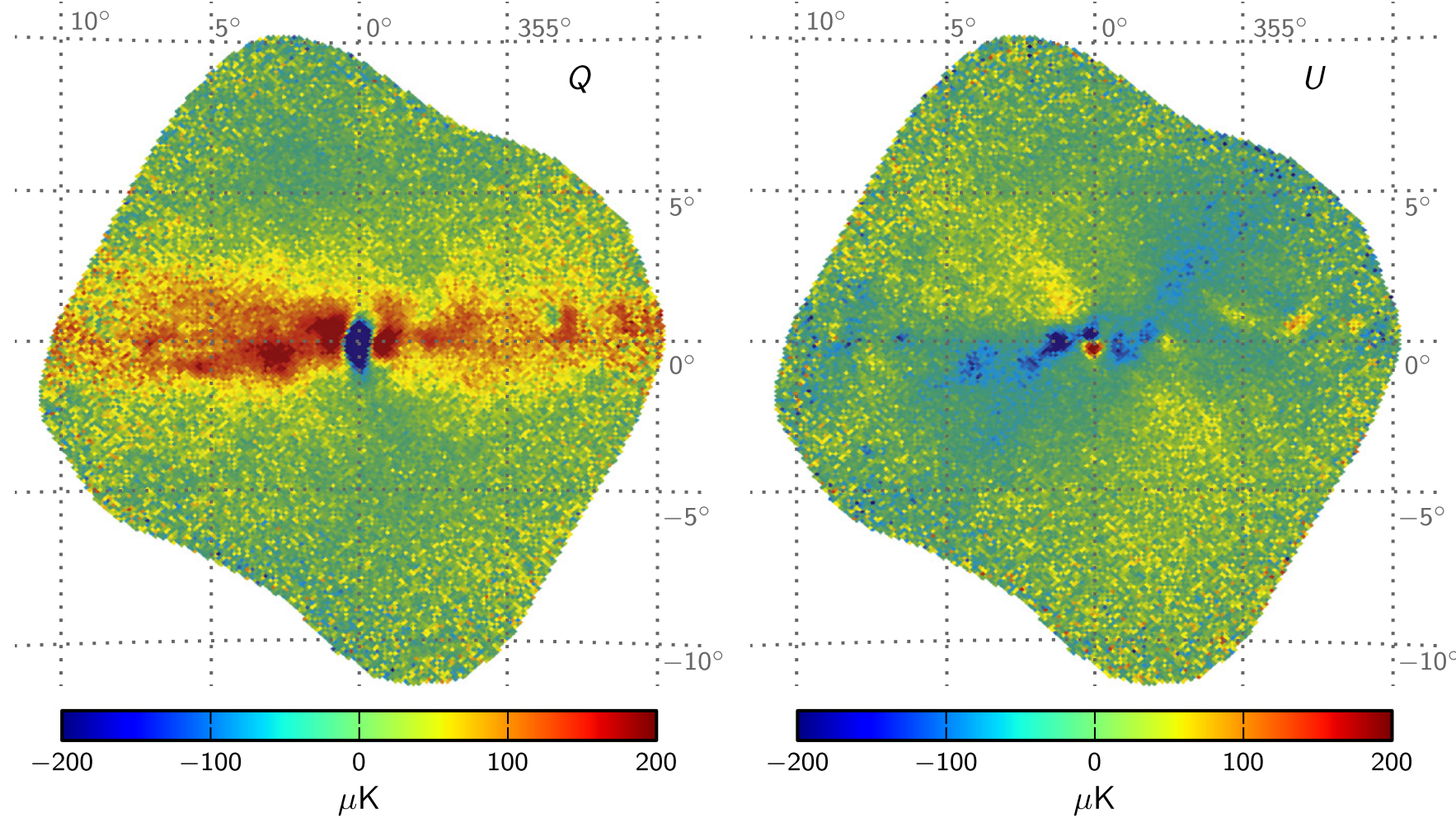

Figure 8. Final co-added QUIET+WMAP Q-band $(43 \mathrm{GHz})$ maps of field G-2 (centered at Galactic coordinates $\left.(l, b)=\left(0^{\circ}, 0^{\circ}\right)\right)$. Using the weight operators defined in Section 2.3, these are expressed as $\boldsymbol{F}_{\mathrm{Q}} \boldsymbol{m}_{\mathrm{Q}}+\boldsymbol{F}_{\mathrm{W}} \boldsymbol{m}_{\mathrm{W}}$. Left and right panels show Stokes $Q$ and $U$, respectively. Grid cell side length is $5^{\circ}$.

WMAP maps in the current set of released maps, but not the Planck maps. Co-addition with Planck, and any other experiment observing the same field, can always be performed later. Similarly, we co-add with the WMAP W1, W2, and W3 differencing assembly maps at $\mathrm{W}$ band, but not $\mathrm{W} 4$, since this particular differencing assembly is known to have significantly worse noise properties than the other three channels (Bennett et al. 2013).

Figure 8 shows the final co-added Q-band G-2 map in Stokes $Q$ and $U$. Comparing this to the filtered maps shown in Figure 4, the most noticeable differences appear, as expected, near the edges of the field, where the QUIET signal-to-noise ratio deteriorates. In the full co-added map, the Stokes $Q$ amplitude remains high along the Galactic plane to the very edge, where it tapers off in the filtered version. Corresponding maps for the other data sets (Q-band G-1 and W-band G-1 and G-2) are shown in Figure 16 in the Appendix.

\section{ASTROPHYSICAL IMPLICATIONS}

The sky maps presented in Section 3 offer a fresh view of astrophysical foregrounds at microwave frequencies. In this section, we compare the co-added QUIET maps to WMAP and Planck, and estimate both the polarized synchrotron spectral index and the Faraday rotation measure in the Galactic center.

\subsection{Visual Inspection of Sky Maps}

In Figures 9 and 10, we compare our maps with the WMAP K-band map (Bennett et al. 2013) and the Planck 2015 map of thermal dust polarization (Planck Collaboration X 2015). All maps have been smoothed with a 30' FWHM Gaussian kernel to reduce instrumental noise. The map of thermal dust is evaluated at $353 \mathrm{GHz}$, whereas the effective frequency of the
$\mathrm{K}$-band map is $22.4 \mathrm{GHz}$ for a synchrotron-like frequency spectrum (Page et al. 2003). The color scale indicates the polarization amplitude, $P$, while the flow pattern traces field lines rotated by $90^{\circ}$ with respect to the measured electric vector position angle (EVPA), corresponding roughly to the magnetic field direction (this correspondence is exact under the assumption of optically thin synchrotron radiation). All plots are generated using an implementation of the Line Integral Convolution algorithm (Cabral \& Leedom 1993) called Alice, provided in the HEALPix package.

Figure 9 shows the Galactic center field G-2. The magnetic field structure in the QUIET Q band (top left panel) shows a correlation with that in the WMAP $\mathrm{K}$-band map (top right panel), showing not only the high quality of the maps, but also indicating that the Q-band sky is dominated by synchrotron emission. According to the Planck 2015 astrophysical baseline model summarized in Figure 51 of Planck Collaboration X (2015), synchrotron emission should dominate over thermal dust emission by about an order of magnitude at $\mathrm{Q}$ band, and our measurements are visually consistent with this picture.

Three individual features are noteworthy in these lowfrequency maps. First, as is well-known (e.g., Page et al. 2007), the orientation of the magnetic field at low Galactic latitudes is parallel to the Galactic plane. Second, the magnetic field lines to the north of the Galactic center form a "U" shape with an opening angle of $\sim 45^{\circ}$. These field lines correspond to the origin of the polarized filaments IX and XIV identified by Vidal et al. (2014a) in the WMAP sky maps. Third, the magnetic field lines in the Galactic center are rotated by an angle of almost $90^{\circ}$ with respect to the Galactic plane in $\mathrm{Q}$ band, while at $\mathrm{K}$ band this angle is about $70^{\circ}$. This is the expected signature of Faraday rotation, and we discuss it quantitatively in Section 4.4. 

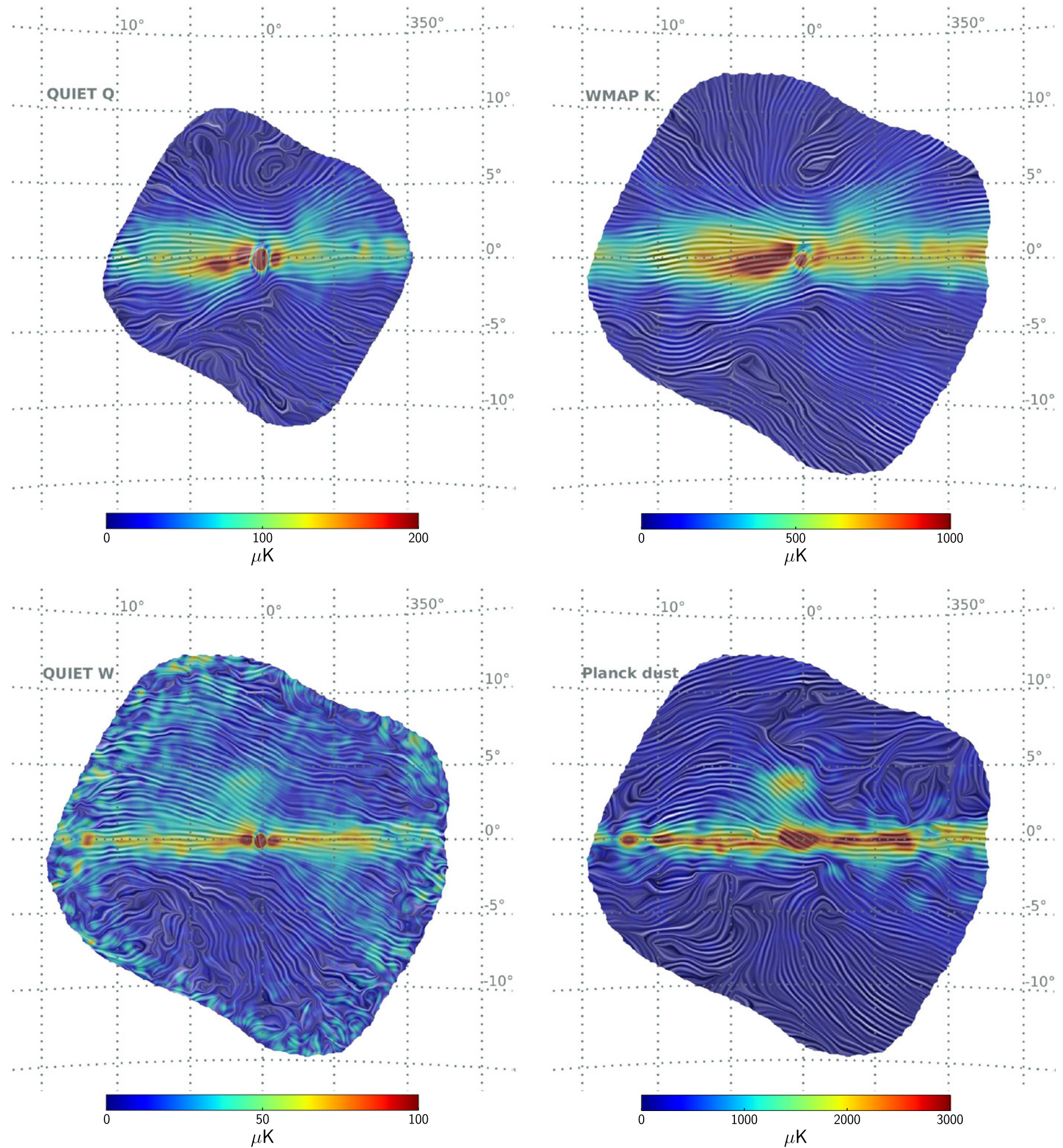

Figure 9. Comparison between the co-added QUIET+WMAP Q-band ( $43 \mathrm{GHz}$, top left) and W-band ( $95 \mathrm{GHz}$, bottom left) maps with the WMAP K-band (23 GHz, top right; Bennett et al. 2013) map and the Planck thermal dust model (evaluated at $353 \mathrm{GHz}$, bottom right; Planck Collaboration X 2015), for field G-2 (centered on Galactic coordinates $(l, b)=\left(0^{\circ}, 0^{\circ}\right)$ ). All plots are generated using the Line Integral Convolution algorithm (Cabral \& Leedom 1993). The color scale indicates polarization amplitude, $P=\sqrt{Q^{2}+U^{2}}$, while the flow stripes indicate magnetic-field orientation, i.e., rotated $90^{\circ}$ with respect to the local polarization orientation.

In the QUIET W-band map (bottom left panel of Figure 9), we also see that the magnetic field is well ordered and parallel to the Galactic plane at low latitudes. Comparison with the Planck thermal dust map (bottom right) suggests that the QUIET map is dominated by dust rather than by synchrotron emission. Based on this qualitative analysis alone, we conclude that the effective polarized foreground minimum must lie between 43 and $95 \mathrm{GHz}$, well separated from either side. Overall, the QUIET measurements are in good agreement with the Planck determination that the foreground minimum lies between 70 and $80 \mathrm{GHz}$ (Planck Collaboration X 2015, in preparation). 

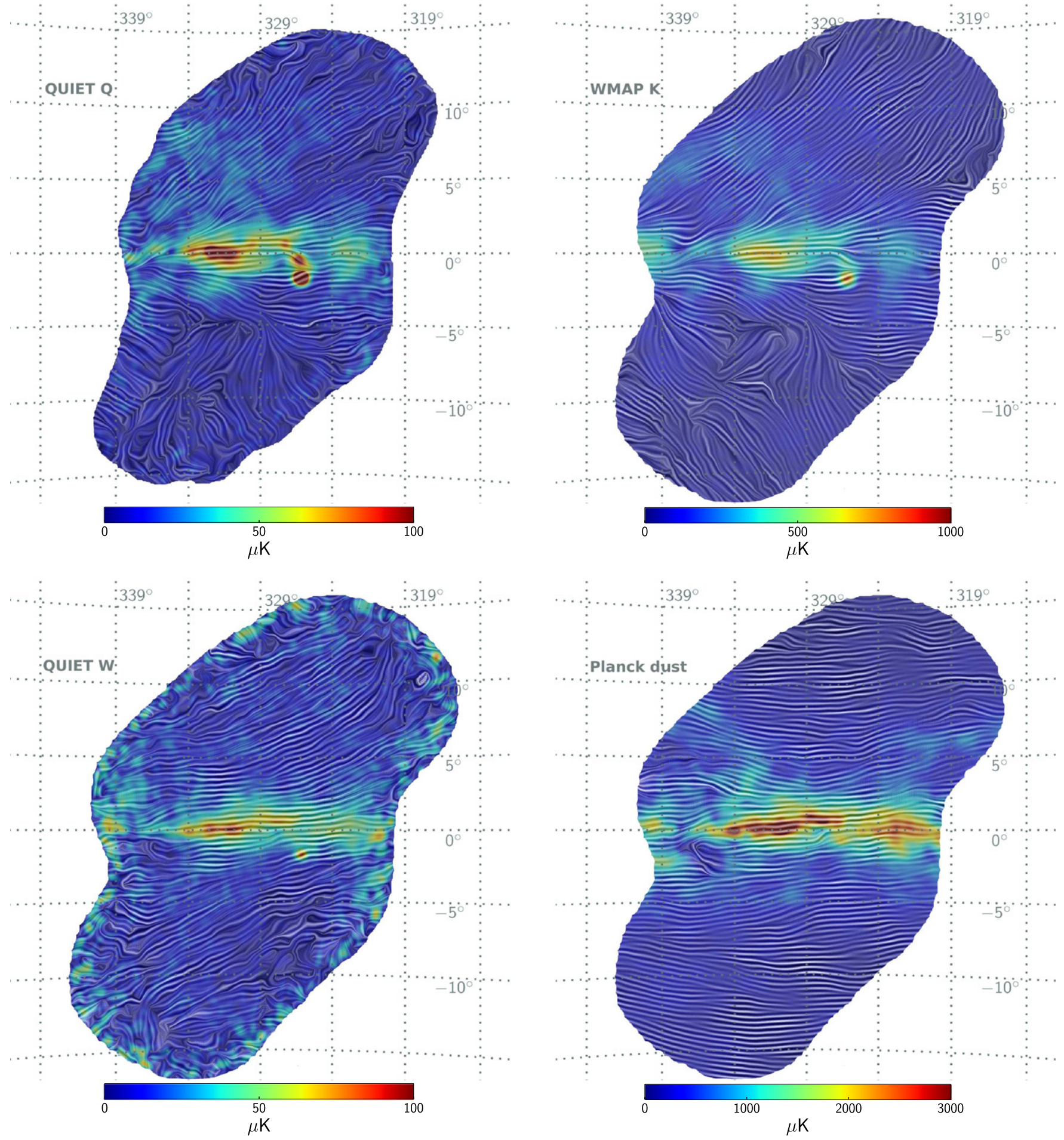

Figure 10. Same as Figure 9, but for G-1.

Figure 10 shows the corresponding information for G-1, the field centered on $(l, b)=\left(329^{\circ}, 0^{\circ}\right)$. Although both maps have lower signal-to-noise ratios than those of G-2, the same qualitative conclusions hold. The Q-band map is clearly dominated by synchrotron emission and the W-band map is dominated by thermal dust emission.

Comparing the QUIET W-band and the Planck thermal dust maps, we note the presence of a bright localized feature in the former at Galactic coordinates $(l, b)=\left(326^{\circ},-2^{\circ}\right)$ with no counterpart in the thermal dust map. We identify this as the supernova remnant (SNR) G326.3-1.8 (MSH 15-56), as discussed by Green (2009). It is a composite SNR that contains a shell with a relatively steep radio spectrum and an interior plerion component with a flat spectrum (Dickel et al. 2000; Weiler \& Sramek 1988). From the $\mathrm{K}, \mathrm{Ka}$, and $\mathrm{Q}$ bands, we estimate its spectral index as $\beta \approx-2.7$, consistent with a (relatively flat spectrum) synchrotron source. This value is steeper than the spectrum of the SNR shell $(\beta=-2.34)$ measured by Dickel et al. (2000) between 0.408 and 14.7 GHz. This new measurement by QUIET might indicate a steepening of the polarized spectrum with frequency. 

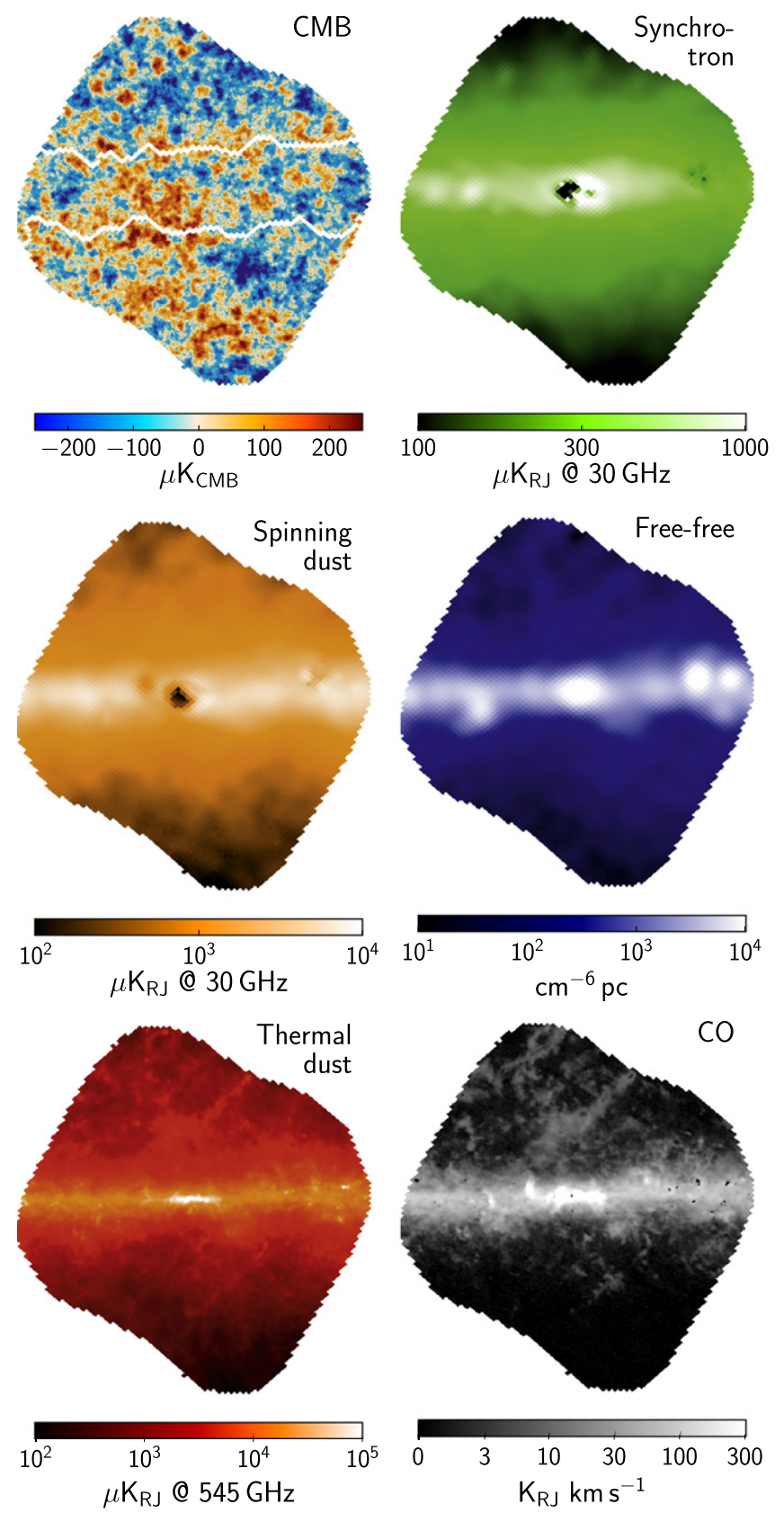

Figure 11. Planck 2015 baseline astrophysical foreground reconstruction in intensity for the G-2 field, as estimated with Commander (Planck Collaboration X 2015, in preparation). From top to bottom and left to right, the panels show (1) CMB, (2) synchrotron, (3) spinning dust, (4) free-free, (5) thermal dust, and (6) $\mathrm{CO} J=2 \rightarrow 1$. The region inside the white boundary in the CMB map has been replaced with a constrained Gaussian realization as part of the Commander algorithm.

Figures 11 and 12 show the Planck baseline temperature reconstruction in our fields, allowing for direct comparison between our maps and individual astrophysical temperature components. From these, we see that the bright G-1 source is indeed recognized as a synchrotron emitter in the Planck model, with little or no counterpart in any other component.

\subsection{Spectral Index of Polarized Emission}

In this section, we determine the effective power-law index between WMAP K band and QUIET Q band, which are heavily dominated by synchrotron emission. All maps are smoothed to
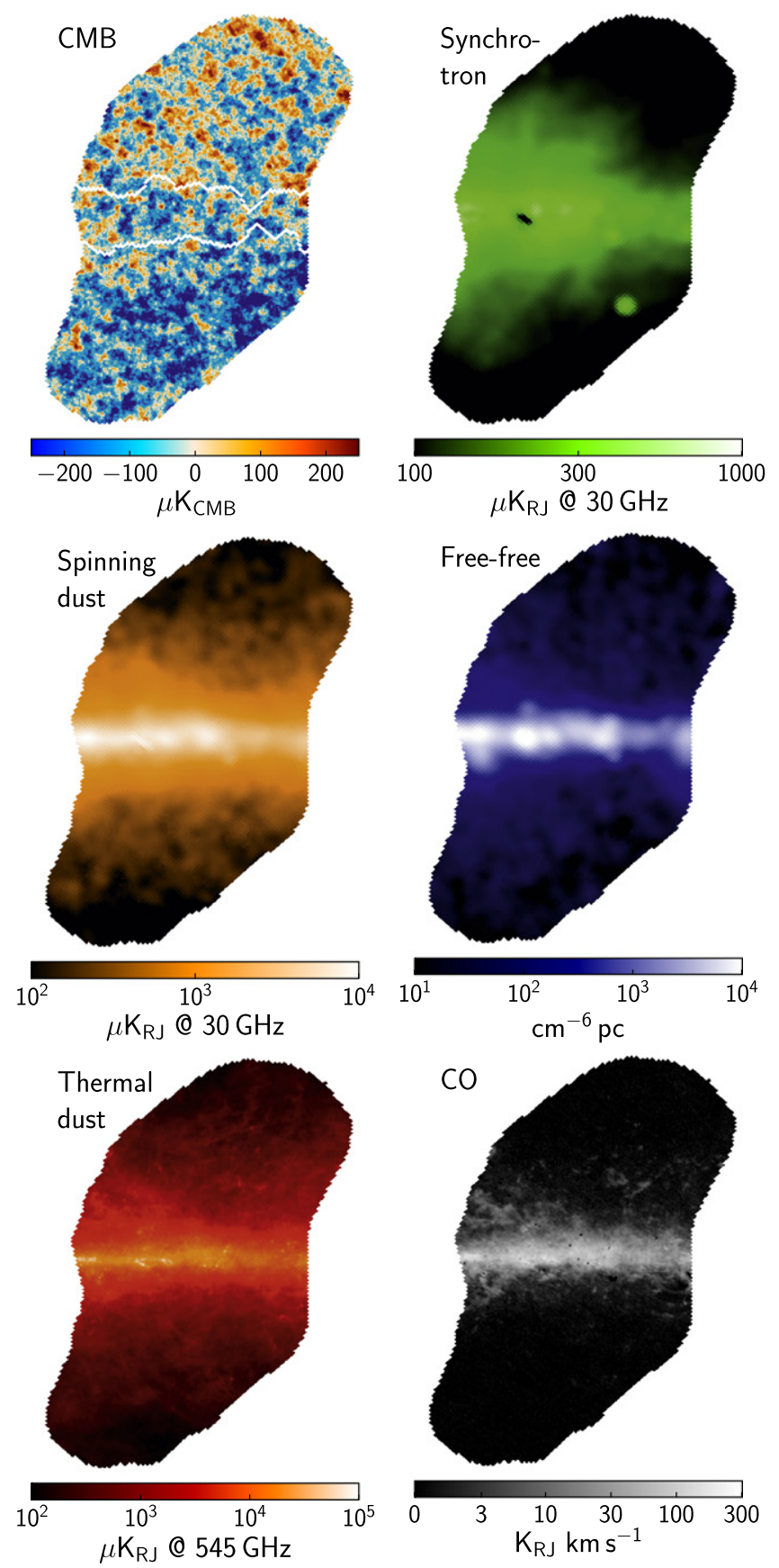

Figure 12. Same as Figure 11, but for field G-1.

a common resolution of $1^{\circ} \mathrm{FWHM}$, slightly larger than the WMAP K-band beam of 53'. All spectral index estimates are derived from the polarization amplitude, $P=\sqrt{Q^{2}+U^{2}}$. The associated bias from instrumental noise is corrected using the asymptotic estimator (Vidal et al. 2014b; Montier et al. 2015), which is a generalization of the estimator first proposed by Wardle \& Kronberg (1974) for the case where the uncertainties $\sigma_{Q}$ and $\sigma_{U}$ are non-identical.

Many studies have reported a significant steepening in the synchrotron spectral index at high Galactic latitudes compared to the Galactic plane (e.g., Kogut et al. 2007; Fuskeland et al. 2014). As a first test, we therefore partition our fields into high $(|b|>2.5)$ and low $(|b| \leqslant 2.5)$ latitudes, and determine 

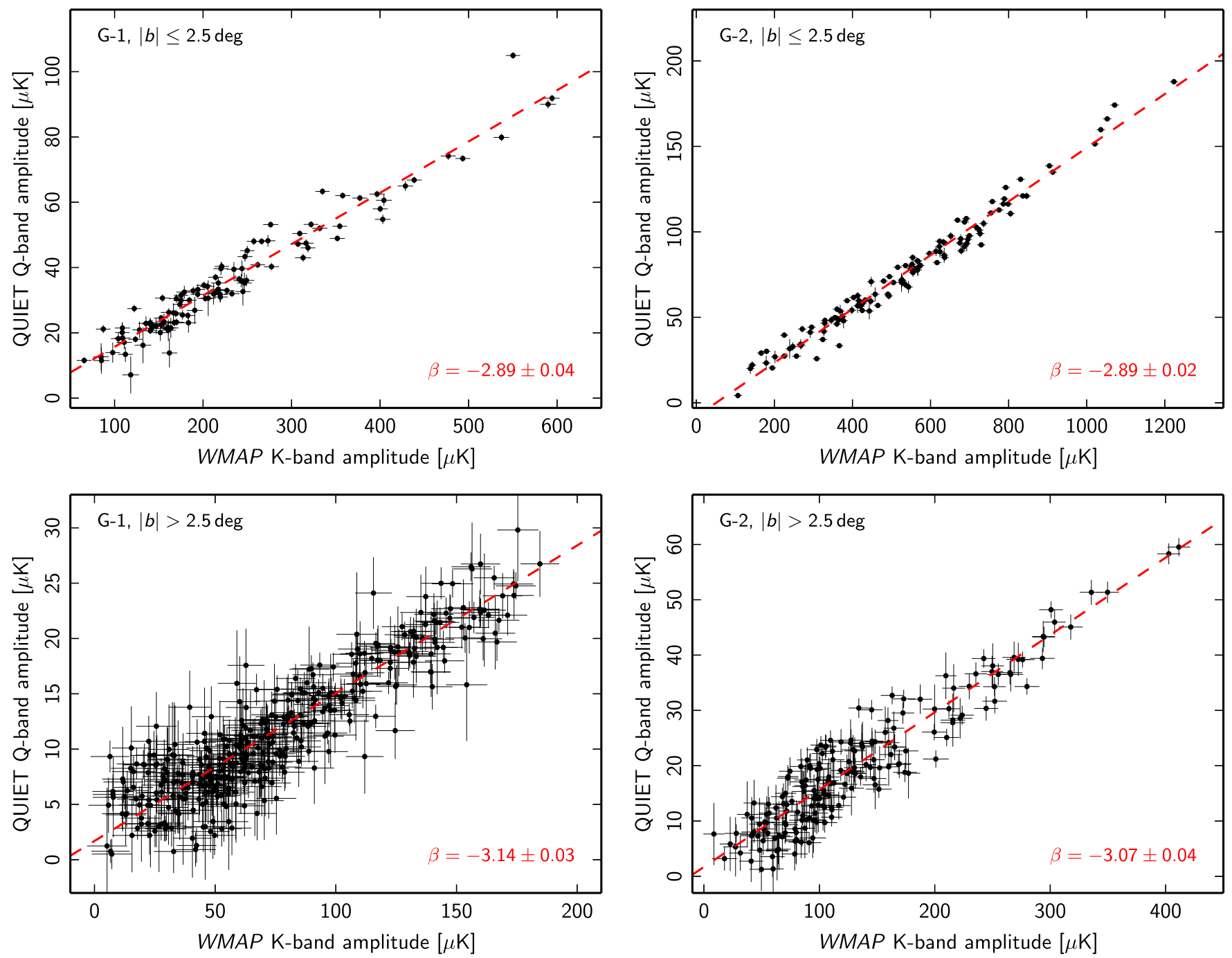

Figure 13. Scatter plots between WMAP K-band ( $23 \mathrm{GHz}$ ) and QUIET Q-band ( $43 \mathrm{GHz}$ ) polarization amplitudes for fields G-1 (left column) and G-2 (right column), considering separately on-plane $(|b| \leqslant 2: 5$; top row) and off-plane $(|b|>2.5$; bottom row) pixels. The dashed red lines indicate the best-fit power-law fit to each data combination.

the spectral indices separately for each region. This calculation is summarized in the scatter plots of Figure 13 (based on polarization amplitudes) between WMAP K band and QUIET Q band for both fields (left and right columns) and low and high latitudes (top and bottom rows). The red dashed lines indicate the best-fit lines, corresponding to the spectral index indicated in each panel. We find results consistent with earlier measurements. In G-2, we derive a Galactic plane spectral index of $\beta=-2.89 \pm 0.02$, which steepens to $\beta=$ $-3.07 \pm 0.04$ at high latitudes. ${ }^{27}$ For G-1, the corresponding numbers are $\beta=-2.89 \pm 0.04$ and $\beta=-3.14 \pm 0.03$. In both cases, the spectral index is about 0.2 steeper off the plane than in the plane.

Including all latitudes in the spectral index evaluation, we find $\beta_{\mathrm{G}-1}=-2.91 \pm 0.01$ and $\beta_{\mathrm{G}-2}=-3.01 \pm 0.01$, as listed in Table 3. These are fully consistent with the values adopted from Fuskeland et al. (2014) $\quad\left(\beta_{\mathrm{G}-1}=-2.93\right.$ and $\beta_{\mathrm{G}-2}=-3.00$; see Section 2.3), derived from WMAP K and $\mathrm{Ka}$ bands, that were used to re-scale the WMAP and Planck maps to the effective QUIET Q-band frequency.

\footnotetext{
${ }^{27}$ All error bars include the uncertainty in the QUIET absolute responsivity.
}

Table 3

Polarized Synchrotron Spectral Index between K and Q Bands ${ }^{\mathrm{a}}$

\begin{tabular}{lcc}
\hline \hline Field & QUIET & WMAP \\
\hline G-1 full & $-2.91 \pm 0.01$ & $-3.02 \pm 0.03$ \\
$|b| \leqslant 2.5$ & $-2.89 \pm 0.04$ & $-3.02 \pm 0.06$ \\
$|b|>2.5$ & $-3.14 \pm 0.03$ & $-3.17 \pm 0.08$ \\
G-2 full & $-3.01 \pm 0.01$ & $-3.02 \pm 0.02$ \\
$|b| \leqslant 2.5$ & $-2.89 \pm 0.02$ & $-2.93 \pm 0.03$ \\
$|b|>2.5$ & $-3.07 \pm 0.04$ & $-3.16 \pm 0.07$ \\
\hline
\end{tabular}

Note.

${ }^{\text {a }}$ Spectral indices are computed between the WMAP $\mathrm{K}$ band and either the QUIET (second column) or the WMAP (third column) Q band.

\subsection{Electric Vector Position Angles}

As noted in Section 4.1 and seen in Figures 9 and 10, the observed orientation of the polarization vector (EVPA) is roughly perpendicular to the Galactic plane in the millimeter wavelength range. Given the assumption of optically thin synchrotron radiation, the Galactic magnetic field is therefore 
Table 4

EVPA as a Function of Galactic Latitude ${ }^{\mathrm{a}, \mathrm{b}}$

\begin{tabular}{|c|c|c|c|c|c|}
\hline \multicolumn{2}{|c|}{ b } & \multicolumn{2}{|c|}{$\chi$ in G-1 } & \multicolumn{2}{|c|}{$\chi$ in G-2 } \\
\hline$+3: 0$ & $+5: 0$ & $30: 0 \pm 0: 3$ & $11: 7 \pm 0: 2$ & $! 3: 5 \pm 0: 3$ & $-20.7 \pm 0.2$ \\
\hline$-1: 0$ & $+1: 0$ & $-0: 8 \pm 0.1$ & $4: 6 \pm 0: 1$ & $7: 0 \pm 0: 1$ & $-4.7 \pm 0.1$ \\
\hline$-3: 0$ & $-1: 0$ & $-5: 3 \pm 0: 2$ & $5: 0 \pm 0.2$ & $10: 1 \pm 0.2$ & $-12: 9 \pm 0.2$ \\
\hline$-5: 0$ & $-3: 0$ & $-9: 9 \pm 0: 4$ & $3: 2 \pm 0: 2$ & $-4: 7 \pm 0.3$ & $-17: 4 \pm 0: 3$ \\
\hline
\end{tabular}

Notes.

${ }^{\text {a }} \chi$ is measured counterclockwise relative to the Galactic north direction.

${ }^{\mathrm{b}}$ Errors are statistical only. See Table 1 for systematic errors.

roughly parallel to the plane. To quantify these alignments, we measure the EVPA, ${ }^{28}$

$$
\chi=\frac{1}{2} \arctan (U / Q)
$$

defined as zero for polarization vectors aligned with Galactic meridians and increasing counter-clockwise in Galactic coordinates. Spatial variations in $\chi$ can be used to constrain models of the Galactic magnetic field (e.g., Jaffe et al. 2013).

In Table 4, we tabulate the mean EVPA as a function of Galactic latitude for both G-1 and G-2, averaging over latitude slabs of $2^{\circ}$ width and masking bright objects. Averaging over the entire fields, the Q-band EVPA is $-1: 9 \pm 0.4$ (stat) $\pm 1: 7$ (syst) for G-1 and $-10: 8 \pm 0: 6$ (stat) $\pm 1: 7$ (syst) for G-2. Similar results were reported by Bierman et al. (2011) for 100 , 150 , and $220 \mathrm{GHz}$ derived from the BICEP observations.

\subsection{Faraday Rotation at the Galactic Center}

The Galactic center patch G-2 includes the brightest polarized source observed by QUIET. Figure 14 shows the QUIET maps of the central region at 43 and $95 \mathrm{GHz}$. The magnetic field of the central source is well aligned and perpendicular to the Galactic plane. In the Galactic plane itself, the magnetic field is parallel to the plane in both bands. At $\mathrm{Q}$ band, a ring with a minimum of polarized emission is seen around the central source. This is due to the cancellation of emission from the source and the Galactic plane with different polarization angles.

The polarization of the Galactic center has been studied in detail at higher angular resolution. Haynes et al. (1992) mapped the region at $8.5 \mathrm{GHz}$ with a beam size of $\sim 2$ !.8 using the Parkes telescope. Tsuboi et al. (1995) also observed this region at $42.5 \mathrm{GHz}$ (similar to QUIET-Q band) with $39^{\prime \prime}$ angular resolution. From these results, it is clear that the dominant source of polarized radio emission originates from the Galactic center "arc." This arc is a very narrow non-thermal filament, $\approx 15^{\prime}$ in length, perpendicular to the Galactic plane, located within a distance of about $20^{\prime}$ from the $\mathrm{Sgr} \mathrm{A}^{*}$ radio source at the Galactic center (see, e.g., Yusef-Zadeh et al. 1984; Pedlar et al. 1989).

We measure the EVPA of the Galactic center arc as a function of frequency from the QUIET, WMAP, and Planck maps, all smoothed to a common resolution of $1^{\circ}$ FWHM, averaging over a $20^{\prime}$ diameter disk centered on the peak of the

\footnotetext{
${ }^{28}$ We remind the reader that QUIET uses the COSMO convention for the Stokes parameters, where Stokes $U$ is replaced by $-U$ to follow the IAU convention.
}

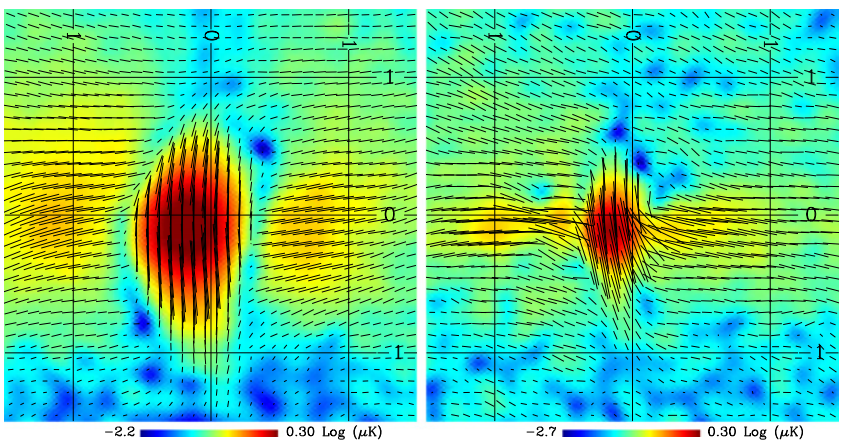

Figure 14. Polarization intensity maps of the Galactic center region seen by QUIET at $43 \mathrm{GHz}$ (left) and $95 \mathrm{GHz}$ (right), at the original angular resolution of $27 ! 3$ and $12^{\prime} .8$ FWHM respectively. The vectors are rotated $90^{\circ}$ with respect to the measured EVPA, indicating the orientation of the magnetic field. Both maps are displayed in Galactic coordinates with a grid size of $1^{\circ}$.

Table 5

Galactic Center Source Polarization Parameters ${ }^{\mathrm{a}}$

\begin{tabular}{|c|c|c|c|}
\hline Band & $\begin{array}{l}\text { Stokes } Q \\
\quad(\mathrm{mK})\end{array}$ & $\begin{array}{c}\text { Stokes } U \\
(\mathrm{mK})\end{array}$ & $\begin{array}{c}\text { Pol angle } \\
\left({ }^{\circ}\right)\end{array}$ \\
\hline QUIET Q & $-1.39 \pm 0.04$ & $0.01 \pm 0.02$ & $90.1 \pm 0.8$ \\
\hline W & $-0.89 \pm 0.06$ & $0.67 \pm 0.03$ & $108.5 \pm 1.3$ \\
\hline$W M A P \mathrm{~K}$ & $-0.44 \pm 0.06$ & $-0.72 \pm 0.03$ & $60.5 \pm 1.4$ \\
\hline $\mathrm{Ka}$ & $-1.06 \pm 0.05$ & $-0.38 \pm 0.03$ & $80.1 \pm 1.2$ \\
\hline $\mathrm{Q}$ & $-1.39 \pm 0.04$ & $-0.17 \pm 0.03$ & $86.6 \pm 0.9$ \\
\hline $\mathrm{V}$ & $-1.62 \pm 0.03$ & $0.26 \pm 0.02$ & $94.6 \pm 0.5$ \\
\hline W & $-0.84 \pm 0.06$ & $0.56 \pm 0.04$ & $106.9 \pm 1.6$ \\
\hline
\end{tabular}

Note.

${ }^{a}$ Values correspond to the mean evaluated inside a $20^{\prime}$ diameter aperture centered at Galactic coordinates $(l, b)=(0 \circ 15,-0 \circ 1)$. Uncertainties in $Q$ and $U$ correspond to the statistical fluctuations in the map, as measured in a larger aperture around the source. See Table 1 for systematic errors.

polarized emission at $(l, b)=(0 \circ 15,-0 \circ 1)$. The resulting values are listed in Table 5, including individual Stokes parameters and EVPAs. The latter are also plotted in Figure 15 as a function of wavelength squared, $\lambda^{2}$. Due to Faraday rotation (e.g., Burn 1966), the observed EVPA is expected to follow $\chi=\mathrm{RM} \lambda^{2}$, where RM is the rotation measure.

Neglecting the W-band data points, we find a tight relation with the expected form, with a best-fit rotation measure of $\mathrm{RM}=-4000 \pm 200 \mathrm{rad} \mathrm{m}^{-2}$, corresponding to a total rotation angle of $\approx 35^{\circ}$ between 23 and $60 \mathrm{GHz}$. Tsuboi et al. (1995) reported a value of $\mathrm{RM}=-3120 \pm 188 \mathrm{rad} \mathrm{m}^{-2}$ between 10 and $42.5 \mathrm{GHz}$ within a $2^{\prime}$ beam centered at the Galactic center arc. The difference between our value and the one from Tsuboi 


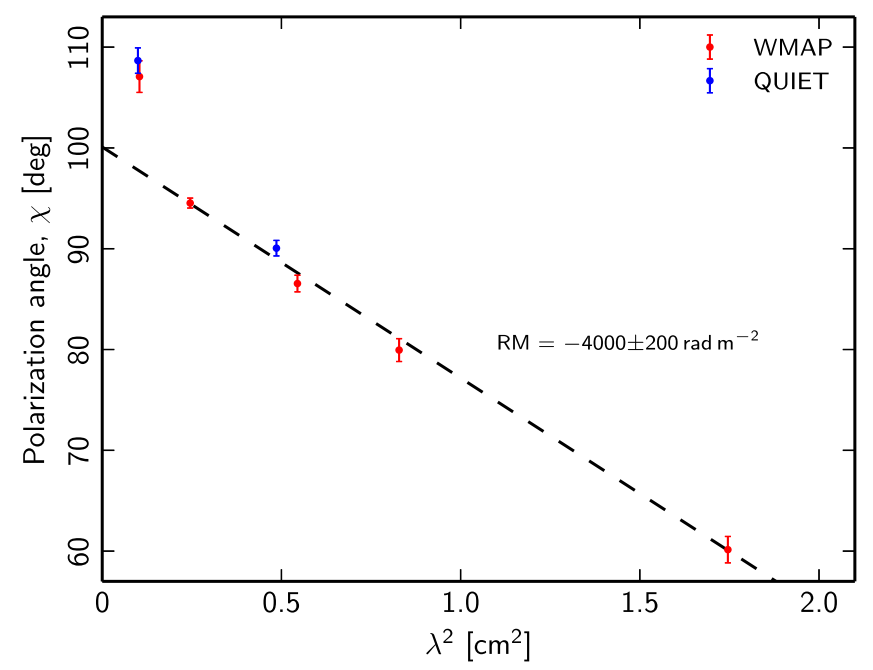

Figure 15. EVPA as a function of wavelength squared for the Galactic center arc for both WMAP (red points), and QUIET (blue points). The dashed line is a linear fit, as expected for a pure foreground Faraday screen resulting in an EVPA $\chi \propto \mathrm{RM} \lambda^{2}$. We exclude the W-band points from the fit; see Section 4.4 for explanation. The best-fit rotation measure is $\mathrm{RM}=-4000 \pm 200 \mathrm{rad} \mathrm{m}^{-2}$.

et al. (1995) could be due to Faraday depolarization at low frequencies, which biases the RM measurements toward lower values (Law et al. 2008). Large absolute values of RM similar to the one we reported here have also been measured close to the Galactic center by Roy et al. (2005).

The EVPAs for the Galactic center arc found in the QUIET sky maps are in good agreement with those reported by WMAP, both at $\mathrm{Q}$ and $\mathrm{W}$ bands. However, the $\mathrm{W}$-band angles differ significantly from the expected Faraday rotation prediction by about $15^{\circ}$. The reason for this is the contribution of dust polarization, which dominates $\mathrm{W}$ band, while synchrotron only dominates below $\sim 60 \mathrm{GHz}$.

\section{SUMMARY}

We have presented polarization measurements of the Galactic plane at 43 and $95 \mathrm{GHz}$, as observed with QUIET between 2008 October and 2010 December. The resulting Galactic maps are the deepest published to date at their respective frequencies, by a factor of two to four at $\mathrm{Q}$ band compared to Planck and WMAP, and by a factor of five to six at W-band compared to WMAP. We find no significant evidence of residual instrumental systematic errors in these high-signalto-noise-ratio maps. We derive a conservative upper limit on temperature-to-polarization leakage of $<0.07 \%$ in the Q-band, translating to a $\lesssim 4 \%$ uncertainty in polarization amplitude at the Galactic center. For comparison, the uncertainty in absolute responsivity is $6 \%$ for $\mathrm{Q}$ band and $8 \%$ for $\mathrm{W}$ band.

Our maps agree very well with corresponding WMAP polarization observations in both $\mathrm{Q}$ and $\mathrm{W}$ bands over the entire field. Accounting for the different effective frequencies and uncertainties in the synchrotron spectral index, we find no compelling evidence for significant systematic differences between the two. At $44 \mathrm{GHz}$, both QUIET and WMAP observe a stronger polarization signal than Planck along the Galactic plane. One potential explanation for this difference is temperature-to-polarization leakage of $\simeq 0.2 \%$ in the Planck data set, which might possibly be related to the nulltest failures for this particular channel already reported by
Planck Collaboration II (2015). Further work is needed to understand these discrepancies in detail.

Exploiting the agreement between QUIET and WMAP, we have computed inverse-noise-variance-weighted averages between the two experiments, combining small-scale information from QUIET with large-scale information from WMAP. The resulting maps are publicly available on LAMBDA, and should prove useful both for experimental consistency checks, as exemplified in this paper, and for understanding the physical properties of polarized foreground emission at microwave wavelengths. In the current paper, we have presented a few examples of such analyses, evaluating the spectral index of synchrotron emission, the mean EVPA near the Galactic plane, and the Faraday rotation measure of the Galactic center source. A key result from this work is robust evidence for true spatial variations in the synchrotron spectral index of diffuse polarized emission along and off the Galactic plane.

Bruce Winstein, who led the QUIET project, died in 2011, soon after observations concluded. The project's success owes a great debt to his intellectual and scientific leadership.

Support for the QUIET instrument and operation comes through the NSF cooperative agreement AST-0506648. Support was also provided by NSF awards PHY-0355328, AST0448909, AST-1010016, and PHY-0551142; KAKENHI 20244041, 20740158, and 21111002; PRODEX C90284; a KIPAC Enterprise grant; and by the Strategic Alliance for the Implementation of New Technologies (SAINT). This work was performed on the Abel cluster, owned and maintained by the University of Oslo and NOTUR (the Norwegian High Performance Computing Consortium). Portions of this work were performed at the Jet Propulsion Laboratory (JPL) and California Institute of Technology, operating under a contract with the National Aeronautics and Space Administration. The Q-band polarimeter modules were developed using funding from the JPL R \& TD program. H.K.E. acknowledges an ERC Starting Grant under FP7. C.D. and M.V. acknowledge support from an ERC Starting Grant (no. 307209). C.D. also acknowledges support from the STFC (U.K.). J.Z. gratefully acknowledges a South Africa National Research Foundation Square Kilometre Array Research Fellowship. L.B. acknowledges support from CONICYT Grant PFB-06. A.D.M. acknowledges a Sloan Fellowship.

PWV measurements were provided by the Atacama Pathfinder Experiment (APEX). We thank CONICYT for granting permission to operate within the Chajnantor Scientific Preserve in Chile, and ALMA for providing site infrastructure support. Field operations were based at the Don Esteban facility run by Astro-Norte. We are particularly indebted to the engineers and technician who maintained and operated the telescope: José Cortés, Cristobal Jara, Freddy Muñoz, and Carlos Verdugo.

In addition, we acknowledge the following people for their assistance in the instrument design, construction, commissioning, operation, and data analysis: Augusto Gutierrez Aitken, Colin Baines, Phil Bannister, Hannah Barker, Matthew R. Becker, Alex Blein, Mircea Bogdan, Alison Brizius, Ricardo Bustos, April Campbell, Anushya Chandra, Sea moon Cho, Sarah Church, Joelle Cooperrider, Mike Crofts, Emma Curry, Maire Daly, Fritz Dejongh, Joy Didier, Greg Dooley, Hans Eide, Pedro Ferreira, Jonathon Goh, Will Grainger, Peter Hamlington, Takeo Higuchi, Seth Hillbrand, Christian Holler, 


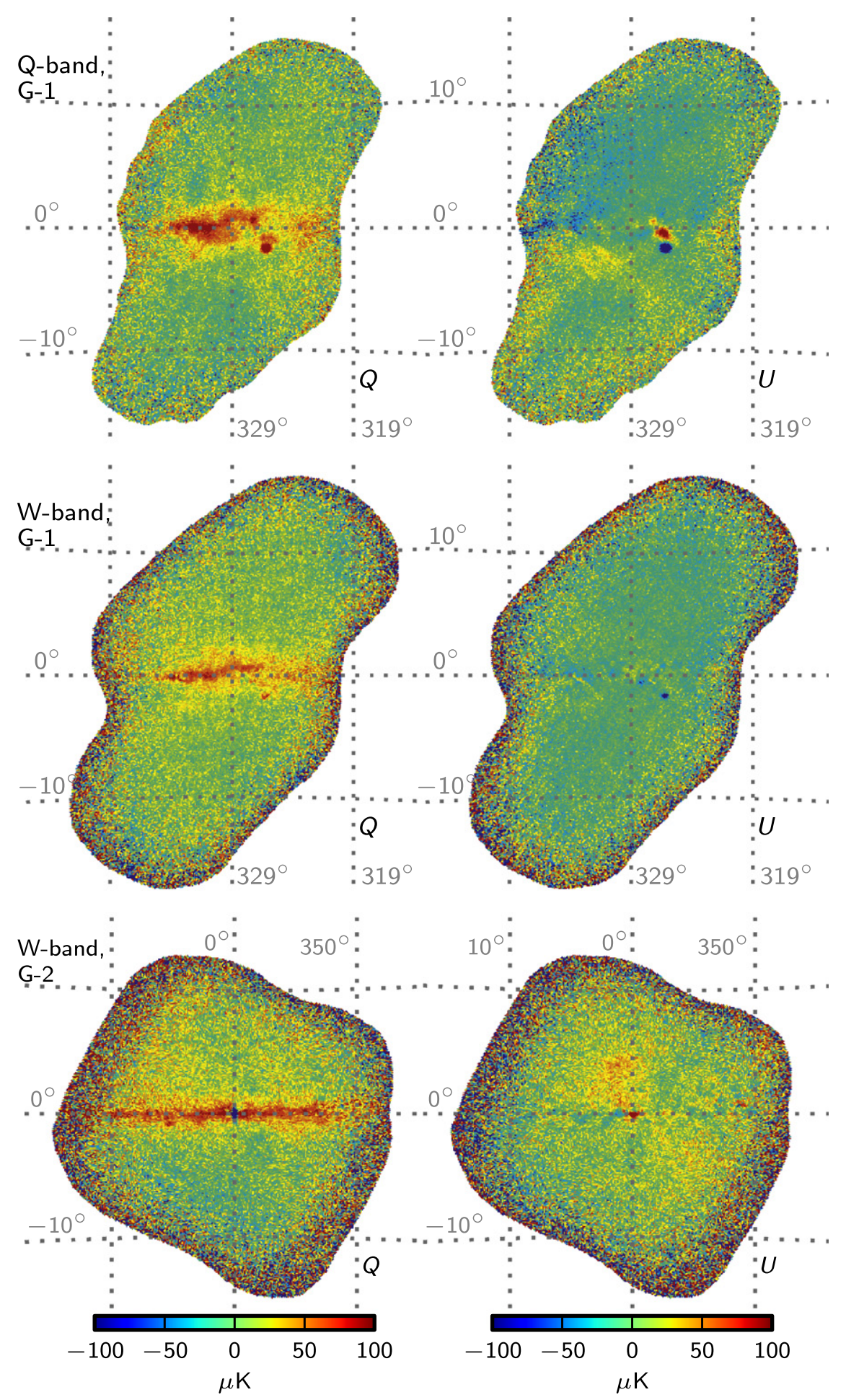

Figure 16. Final co-added QUIET $+W M A P$ maps. The top row shows the Q-band $(43 \mathrm{GHz})$ result for field G-1 $\left(\right.$ centered on Galactic coordinates $\left.(l, b)=\left(329^{\circ}, 0^{\circ}\right)\right)$ Using the weight operators defined in Section 2.3, these maps are expressed as $\boldsymbol{F}_{\mathrm{Q}} \boldsymbol{m}_{\mathrm{O}}+\boldsymbol{F}_{\mathrm{W}} \boldsymbol{m}_{\mathrm{W}}$. The middle and bottom rows show the equivalent maps for W band $(95 \mathrm{GHz})$, both fields. The left and right columns show Stokes $Q$ and $U$, respectively. The grid cell width is $10^{\circ}$.

Ben Hooberman, Kathryn D. Huff, William Imbriale, Koji Ishidoshiro, Norm Jarosik, Pekka Kangaslahti, Dan Kapner, Oliver King, Eiichiro Komatsu, Jostein Kristiansen, Donna Kubik, Richard Lai, David Leibovitch, Kelly Lepo, Siqi Li, Martha Malin, Jorge May, Mark McCulloch, Jeff McMahon, Steve Meyer, Oliver Montes, David Moore, Makoto Nagai, Hogan Nguyen, Glen Nixon, Ian O’Dwyer, Gustavo Orellana,
Stephen Osborne, Heather Owen, Stephen Padin, Felipe Pedreros, Ashley Perko, Joey Richards, Alan Robinson, Jacklyn Sanders, Dale Sanford, Yunior Savon, Kendrick Smith, Mary Soria, Alex Sugarbaker, David Sutton, Keith Vanderlinde, Liza Volkova, Ross Williamson, Edward Wollack, Stephanie Xenos, Octavio Zapata, Mark Zaskowski, and Joe Zuntz. 

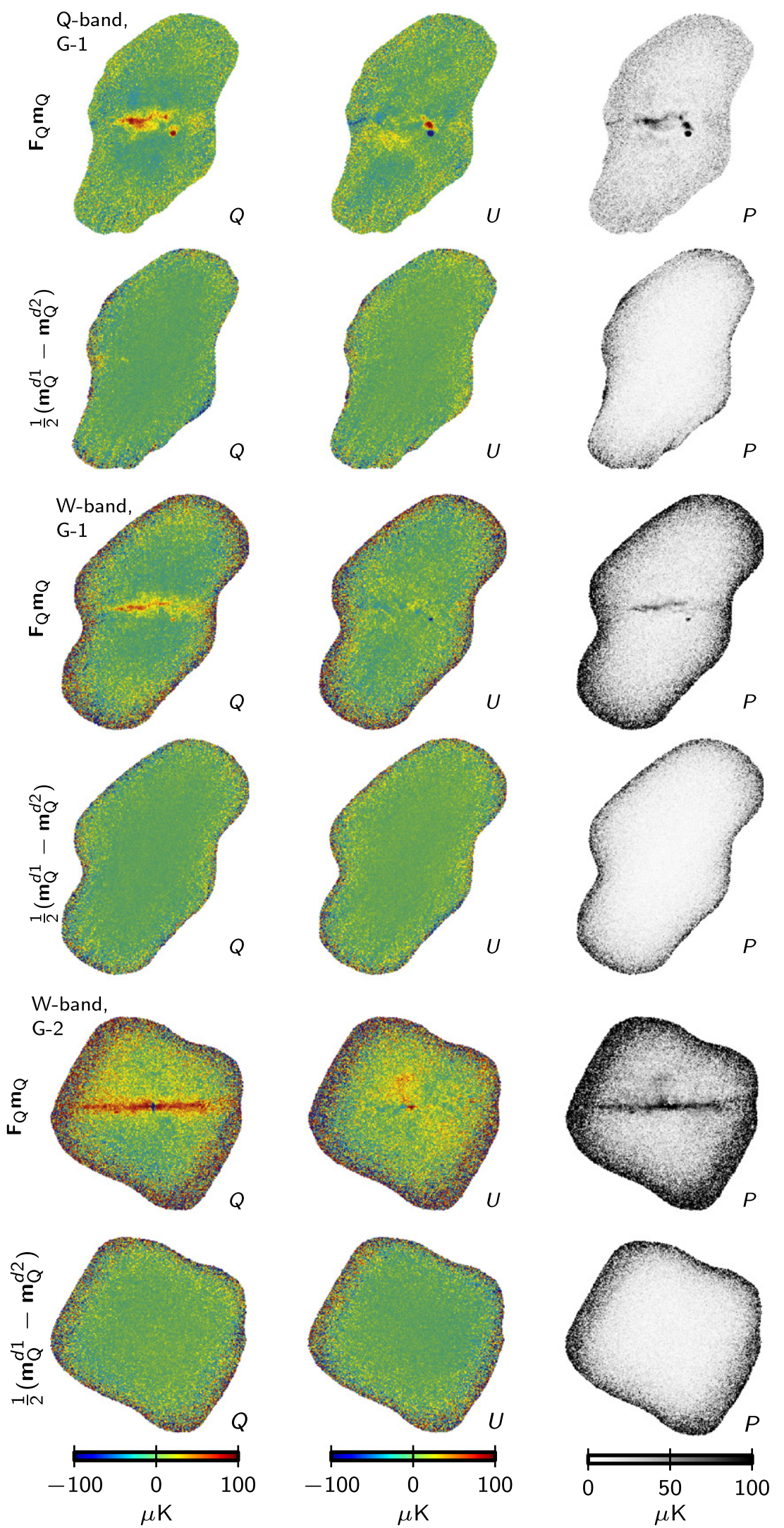

Figure 17. Inverse-noise-variance-weighted QUIET maps and deck-split half-difference maps. Columns show, from left to right, Stokes $Q$, Stokes $U$, and polarization amplitude $P$. The first row shows the QUIET map $\boldsymbol{m}_{\mathrm{O}}$, for the G-1 field (centered on Galactic coordinates $(l, b)=\left(329^{\circ}, 0^{\circ}\right)$ ) in Q band $(43 \mathrm{GHz})$, filtered to only contain the small-scale modes observable by QUIET, as determined by the QUIET weighting operator $\boldsymbol{F}_{\mathrm{Q}}$ defined in Section 2.3. The second row shows the corresponding deck-split half-difference map, $\frac{1}{2}\left(\boldsymbol{m}_{Q}^{d 1}-\boldsymbol{m}_{Q}^{d 2}\right)$. The following rows show the equivalent pairs of maps for both fields in $\mathrm{W}$ band $(95 \mathrm{GHz})$. 

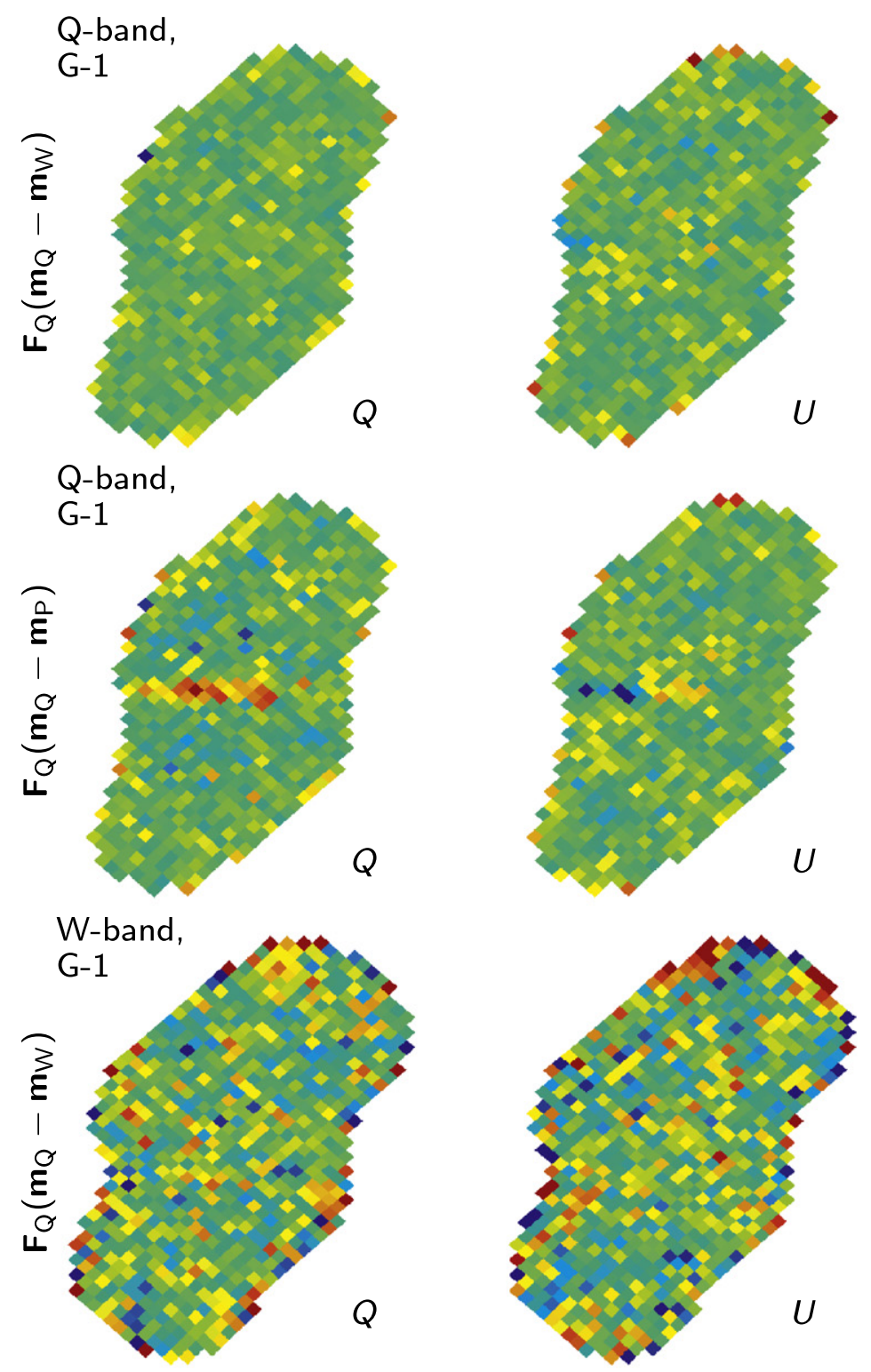

W-band, G-2
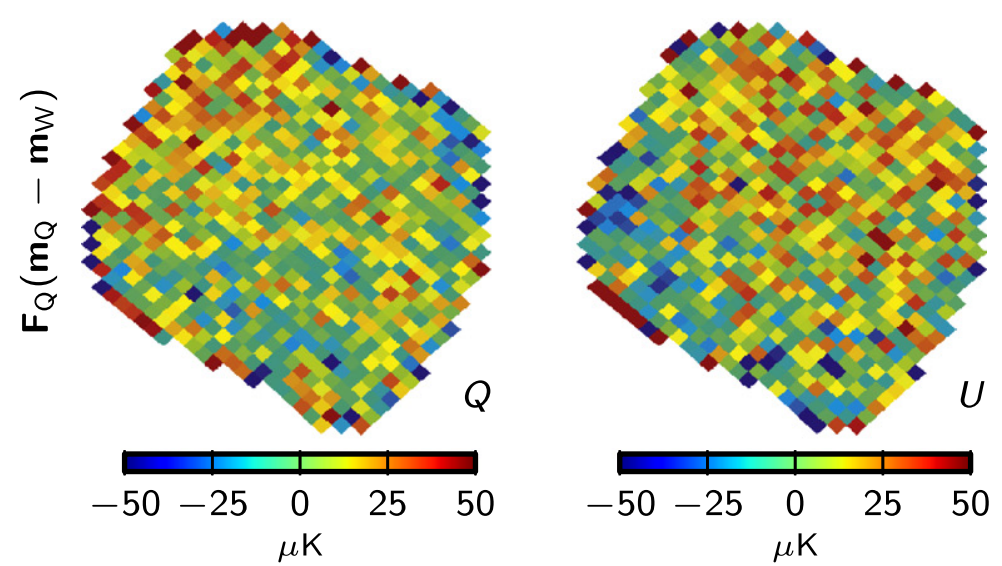

Figure 18. Pairwise differences of QUIET and WMAP or Planck maps, downgraded to HEALPix $N_{\text {side }}=64$ to suppress noise. All maps are weighted using the QUIET weight operator $\boldsymbol{F}_{\mathrm{Q}}$, defined in Section 2.3, retaining only the small-scale modes observed by QUIET in the differenced maps. The top row shows QUIETWMAP for field G-1 (centered on Galactic coordinates $(l, b)=\left(329^{\circ}, 0^{\circ}\right)$ ) in Q band (43 GHz). The second row shows the corresponding difference of QUIETPlanck, while the third and fourth rows show QUIET-WMAP for W band $(95 \mathrm{GHz})$, both fields. Columns show, from left to right, Stokes $Q$ and Stokes $U$. 
Some of the results in this paper have been derived using the HEALPix package.

\section{APPENDIX SUPPORTING FIGURES}

In Section 3, we presented both the raw QUIET and the coadded QUIET+WMAP Q-band sky maps as derived for the Galactic center field, G-2. We also showed an internal consistency test for this field between QUIET, WMAP, and Planck, in the form of difference maps. In this appendix, we show corresponding plots for the remaining three data combinations, namely the Q-band G-1 and W-band G-1 and G-2 fields. All of the main conclusions remain unchanged compared to the original discussion, and the following plots are reproduced for completeness and reference purposes alone. Null-map statistics for all fields and best-fit linear regression slopes for the Q-band data are listed in Table 1.

Figure 16 shows the final co-added QUIET $+W M A P$ Stokes $Q$ and $U$ parameter maps for Q-band G-1 (top row), W-band G-1 (middle row), and W-band G-2 (bottom row), corresponding to Figure 8 for the Q-band G-2 field in the main text. Computing the polarization amplitudes and EVPAs from these leads to the maps shown in Figures 9 and 10.

Figure 17 shows the (filtered) QUIET contributions to the co-added sky maps and the deck-split half-difference maps for each field combination, corresponding to Figure 4 in the main text. No significant residuals are seen in any of these difference maps. The Q-band G-2 case discussed in the main text therefore represents a conservative worst-case scenario with respect to temperature-to-polarization leakage.

Figure 18 shows the difference maps between QUIET, WMAP, and Planck, all downgraded to $N_{\text {side }}=64$ as in Section 3.2. Differences with respect to Planck are only evaluated for the Q-band G-1 field, since Planck does not provide a polarized W-band map at this time. As for the G-2 field, we note a significant positive residual with respect to Planck in G-1, while no significant residuals are seen with respect to WMAP in either case. Finally, it could be noted that the W-band difference maps have a higher noise level. This is due to the high noise rms in the WMAP W-band maps, as listed in Table 1.

\section{REFERENCES}

Bennett, C. L., Larson, D., Weiland, J. L., et al. 2013, ApJS, 208, 20 BICEP2 Collaboration, Ade, P. A. R., Aikin, R. W., et al. 2014, PhRvL, 112, 241101

BICEP2 Collaboration, Ade, P. A. R., Aikin, R. W., et al. 2015, PhRvL, 114 101301

BICEP2/Keck Array and Planck Collaborations 2015, PhRvL, submitted (arXiv:1502.00612)

Bierman, E. M., Matsumura, T., Dowell, C. D., et al. 2011, ApJ, 741, 81

Bond, J. R., Jaffe, A. H., \& Knox, L. 1998, PhRvD, 57, 2117

Burn, B. J. 1966, MNRAS, 133, 67

Cabral, B., \& Leedom, L. C. 1993, in SIGGRAPH, Proc. 20th Annual Conf. on Computer Graphics and Interactive Techniques (New York: ACM), 263
Carretti, E., Gaensler, B., Staveley-Smith, L., et al. 2009, S-band Polarization All Sky Survey (S-PASS), ATNF Proposal

Chiang, H. C., Ade, P. A. R., Barkats, D., et al. 2010, ApJ, 711, 1123

de Oliveira-Costa, A., Tegmark, M., Gaensler, B. M., et al. 2008, MNRAS, 388,247

Dickel, J. R., Milne, D. K., \& Strom, R. G. 2000, ApJ, 543, 840

Finkbeiner, D. P., Davis, M., \& Schlegel, D. J. 1999, ApJ, 524, 867

Fuskeland, U., Wehus, I. K., Eriksen, H. K., \& Næss, S. K. 2014, ApJ, 790, 104

García, P., Bronfman, L., Nyman, L.-Å., Dame, T. M., \& Luna, A. 2014, ApJS, 212, 2

Gorski, K. M., Hivon, E., Banday, A. J., et al. 2005, ApJ, 622, 759

Green, D. A. 2009, BASI, 37, 45

Haynes, R. F., Stewart, R. T., Gray, A. D., et al. 1992, A\&A, 264, 500

Hivon, E., Gorski, K. M., Netterfield, C. B., et al. 2002, ApJ, 567, 2

Ichiki, K. 2014, PTEP, http://ptep.oxfordjournals.org/content/2014/6/ 06B109.full.pdf

Jaffe, T. R., Ferrière, K. M., Banday, A. J., et al. 2013, MNRAS, 431, 683

Keskitalo, R., Ashdown, M. A. J., Cabella, P., et al. 2010, A\&A, 522, A94

King, O. G., Copley, C., Davies, R., et al. 2010, Proc. SPIE, 7741, 1

King, O. G., Jones, M. E., Blackhurst, E. J., et al. 2014, MNRAS, 438, 2426

Kogut, A., Dunkley, J., Bennett, C. L., et al. 2007, ApJ, 665, 355

Law, C. J., Yusef-Zadeh, F., Cotton, W. D., \& Maddalena, R. J. 2008, ApJS, 177,255

Liddle, A. R., \& Lyth, D. H. 2000, Cosmological Inflation and Large-Scale Structure (Cambridge: Cambridge Univ. Press)

MacTavish, C. J., Ade, P. A. R., Bock, J. J., et al. 2006, ApJ, 647, 799

Montier, L., Plaszczynski, S., Levrier, F., et al. 2015, A\&A, 574, A136

Page, L., Barnes, C., Hinshaw, G., et al. 2003, ApJS, 148, 39

Page, L., Hinshaw, G., Komatsu, E., et al. 2007, ApJS, 170, 335

Pedlar, A., Anantharamaiah, K. R., Ekers, R. D., et al. 1989, ApJ, 342, 769

Petrolini, A. 2014, AmJPh, 82, 1178

Planck Collaboration I 2014, A\&A, 571, A1

Planck Collaboration VII 2011, A\&A, 536, A7

Planck Collaboration XV 2014, A\&A, 571, A15

Planck Collaboration XVI 2014, A\&A, 571, A16

Planck Collaboration I 2015, A\&A, submitted (arXiv:1502.01582)

Planck Collaboration II 2015, A\&A, submitted (arXiv:1502.01583)

Planck Collaboration VIII 2015, A\&A, submitted (arXiv:1502.01587)

Planck Collaboration X 2015, A\&A, submitted (arXiv:1502.01588)

Planck Collaboration XI 2015, A\&A, submitted (arXiv:1507.02704)

Planck Collaboration XIII 2015, A\&A, submitted (arXiv:1502.01589)

QUIET Collaboration, Araujo, D., Bischoff, C., et al. 2012a, ApJ, 760, 145

QUIET Collaboration, Bischoff, C., Brizius, A., et al. 2011, ApJ, 741, 111

QUIET Collaboration, Bischoff, C., Brizius, A., et al. 2012b, arXiv:1207.5562

QUIET Collaboration, Huffenberger, K. M., Araujo, D., et al. 2014, arXiv:1412.1111

Readhead, A. C. S., Myers, S. T., Pearson, T. J., et al. 2004, Sci, 306, 836

Roy, S., Rao, A. P., \& Subrahmanyan, R. 2005, MNRAS, 360, 1305

Rubiño-Martín, J. A., López-Caraballo, C. H., Génova-Santos, R., \& Rebolo, R. 2012, AdAst, 2012, 40

Sievers, J. L., Achermann, C., Bond, J. R., et al. 2007, ApJ, 660, 976

Tegmark, M. 1997, ApJL, 480, L87

Tsuboi, M., Kawabata, T., Kasuga, T., Handa, T., \& Kato, T. 1995, PASJ, 47,829

Vidal, M., Dickinson, C., Davies, R. D., \& Leahy, J. P. 2014a, MNRAS, in press (arXiv:1410.4438)

Vidal, M., Leahy, J. P., \& Dickinson, C. 2014b, MNRAS, submitted (arXiv:1410.4436)

Wardle, J. F. C., \& Kronberg, P. P. 1974, ApJ, 194, 249

Weiler, K. W., \& Sramek, R. A. 1988, ARA\&A, 26, 295

Woodbury, M. A. 1950, Inverting Modified Matrices, Statistical Research Group, Memo. Rep. No. 42 (Princeton, NJ: Princeton University), 4

Yusef-Zadeh, F., Morris, M., \& Chance, D. 1984, Natur, 310, 557 